\title{
A Self-adjusting Rate Adaptation Scheme with Good Fairness and Smoothness Properties
}

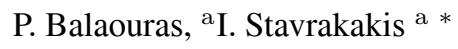

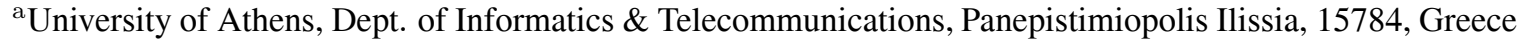

\begin{abstract}
A novel feedback-based rate adaptation scheme is introduced and investigated in this paper. Its main innovative characteristic is the modulation of the rate increment by the distance between a flow's present rate and an assumed targeted maximum rate as dictated by the associated application. The previous along with the shaping of the rate decrement by the reported flow's losses are responsible for a dynamic and self-adjusting behavior that is shown to improve convergence to fairness, the oscillatory behavior of the rate and the induced packet losses when compared with the basic Additive Increase Multiplicative Decrease (AI/MD) scheme. Numerical results illustrate the good properties and intrinsic advantages of the proposed scheme both under the considered modeling assumptions, as well as under more real networking conditions by employing the ns-2 simulator. A brief comparison of the proposed scheme with the TCP-compatible schemes TFRC, IIAD and the non-AI/MD schemes AIPD, LIMPD, is included as well. Because of the aforementioned induced behavior and assumed flow's characteristics (min and max rates), the proposed congestion control scheme seems to be appropriate for regulating the rate of streaming applications.
\end{abstract}

\section{Introduction}

Congestion control schemes are necessary in order for shared resource networks to avoid or overcome congestion. Such a scheme - that is based on an Additive Increase Multiplicative Decrease (AI/MD) algorithm [1,3], a linear control algorithm - is employed in today's Internet by the Transmission Control Protocol (TCP) [2] and is largely responsible for its robustness and stability. TCP has been designed - and successfully used - for unicast reliable data transfer over low bandwidth lines. One of its primary goals for fast recovery from congestion is achieved by halving ( multiplicative decrease factor $b_{\mathrm{D}}$ equal to 0.5 ) the transmission rate upon congestion. The rate halving affects the TCP flows' smoothness, making TCP and generally all $\mathrm{AI} / \mathrm{MD}$ schemes with a small value of $b_{\mathrm{D}}$ - unsuitable for the continuous media (CM) streaming applications. Furthermore, the TCP's retransmission mechanism introduces typically unacceptable end-to-end delay and delay variation for $\mathrm{CM}$ applications. The requirement for the timely delivery prohibits the recovery of the lost packets through

\footnotetext{
*Work supported in part by the General Secretariat for Research and Technology of Greece under a 2002-4 Joint Greek-Italian Scientific and Technological Cooperation Programme and by the Special Account for Research Grants of the National and Kapodistrian University of Athens .
}

a retransmission scheme as the recovery mechanism typically fails to meet the decoding deadlines [21,22]. This is the reason why the User Datagram Protocol (UDP), that lacks a retransmission mechanism is mainly used to support the $\mathrm{CM}$ streaming applications in conjunction with the Real-time Transport Protocol (RTP) and Real-time Transport Control Protocol (RTCP) [5]. Applications that use UDP as transport protocol should also implement an end-to-end congestion control scheme, like TCP, to retain the stability of the Internet. Otherwise, all supported applications will suffer and eventually the network will collapse [6]. Alternative approaches focus on providing for a smoother adaptation while achieving a similar throughput to a TCP flow $[4,16,20,23]$.

This work is motivated by the need of CM flows to smoothly adapt their rate toward the fair share in a dynamic environment where new (existing) $\mathrm{CM}$ flows are initiated (terminated). We take into consideration the following characteristics/constraints of the encoders in order to improve the adaptation behavior of the CM flows:

(a) the existence of a minimum transmission rate $m$ that corresponds to the lower acceptable perceptual quality for the end user;

(b) the existence of a maximum transmission rate $M$ that may correspond to one of the following: (i) the maximum rate of the encoder, (ii) a high level 
of perceptual quality, over which the gain of the perceived quality is relatively low, (iii) the maximum rate that the receiver could receive due to link or decoding capabilities constraints;

(c) the fact that commercial CM streaming applications are rate-based and RTP/RTCP-based, therefore the use of RTCP reports as a feedback mechanism eliminates the need for an additional mechanism and enables the use of continuous (as opposed to binary) feedback (i.e., packet loss rate) in the decrease policy;

(d) the encoder's inability to adjust its encoding rate at a high frequency (i.e., once every round trip time (RTT)) and instantaneously, resulting in intervals between successive adaptation point of larger time scale than that of RTT.

Specifically, we explore in the present work a sender-driven rate-based adaptation scheme that exploits the knowledge of (a) a pair of minimum and maximum allowable rates $(m, M)$ in the increase policy, which are considered to be common to all flows in this paper, and (b) the packet loss rate in the decrease policy. The increase function depends on the distance between the current rate and a maximum allowable rate (Distance Weighted Additive Increase (DWAI) policy). The use of the minimum and maximum allowable rates allow for a variable rate increment that is maximized when the flow's rate reaches the minimum allowable rate and minimized when the flow's rate reaches the maximum allowable rate. Therefore, since lower rate flows are more aggressive than higher rate flows, the proposed increase policy converges faster to the fair share than the basic AI which has a neutral impact to the fairness. We use the reported packet loss rate in the decrease policy (Loss rate Dependent Multiplicative Decrease (LDMD) policy) since schemes based on loss dependent decrease policies, such as those presented in $[10,29,18]$, are capable of adjusting the flow's rate according to the loss rate and present a smoother adaptation behavior than the basic multiplicative decrease policy. The new rate shaping is based on the current "effective sending rate" $((1-$ current loss rate $) \times$ current rate $)$ scaled by a factor $d$ (new rate $=d \times($ effective sending rate). We show that the proposed LDMD policy has interesting properties that are not encountered in basic $\mathrm{AI} / \mathrm{MD}$ and non $\mathrm{AI} / \mathrm{MD}$ schemes $\mathrm{AI} / \mathrm{PD}$ and LIMDH $[10,29,18]$ (See simulation results and related work).

The proposed scheme is not TCP-compatible. The considered network environment is, thus, similar to the forthcoming one based on the DiffServ architecture [17], in which flows of common requirements (e.g., CM flows) will probably be isolated from flows with other requirements (e.g., TCP data flows). Even in the context of a DiffServ environment, CM flows should be elastic (rate adaptive) since the total load may exceed the available capacity [7]. The proposed scheme is directly applicable to a DiffServ environment supporting CM applications.

In the proposed scheme, the packet loss rate is reported by a feedback mechanism based on RTCP. RTCP Receiver Reports (RR) inform the sender every 5 seconds about the packet losses occurred over the last time interval. Note that the interval between two successive feedbacks is of a larger time scale than RTTs. This has the advantage that the packet loss rate does not need to be estimated from the acknowledgements, as required in schemes in which each packet is acknowledged $[4,15,23]$, but can be accurately calculated by the sender since the number of transmitted packet are known and the number of packets lost over the last interval are reported by the receiver. The disadvantage of a RTCP-based feedback mechanism maybe that the response to congestion or bandwidth availability, which depends on the aforementioned interval, is slower which might result in more packet losses. The latter effect is mitigated by (a) the fact that CM flows tolerate losses provided they are low, and (b) the possible usage of a packet marking policy in the routers instead of a packet dropping policy considering the losses as virtual ones. These facts suggest that it is more meaningful to attempt to improve the smoothness and convergence to fairness characteristics of the rate adaptation process, rather than improve the packet loss rate. This does not mean that the packet loss rate, throughput or network utilization are necessary worse under the proposed scheme. We show that RTCP-based schemes are capable of achieving better smoothness than the window-based TCP-compatible schemes TFRC [16] and IIAD [20].

This paper is organized as follows. In Section 2 , a novel congestion control scheme is presented that takes into consideration the distance between the flow's current rate and its predetermined upper bound, 
as well as the packet loss rate reported by the feedback. In Section 3, the convergence to efficiency and fairness of the presented scheme is proved. In Section 4, the adaptation behavior of the presented scheme is analyzed and quantified in terms of responsiveness to BW availability, adaptation behavior, oscillation sizes, packet loss rate and throughput. The improved behavior of the proposed scheme over the basic AI/MD scheme in terms of convergence speed to fairness, smoothness and packet loss rates is discussed in Section 5. This better behavior is demonstrated through a set of simulation results in Section 6. Comparison of the DWAI/LDMD scheme to the TFRC, IIAD, AI/PD and LIMDH schemes is included as well. Section 7 discusses related work. Finally, the conclusions are presented in the last section.

\section{Description of the Distance Weighted Additive Increase and Loss Rate Dependent Multiplica- tive Decrease Scheme}

In this section, a novel congestion control scheme - referred to as the DWAI/LDMD scheme - is introduced. The major characteristics of this scheme are: (a) the rate increment depends on the distance between the current rate and a maximum allowable rate (Distance Weighted Additive Increase (DWAI) policy); (b) the rate decrease depends on the actual value of the reported packet loss rate (Loss rate Dependent Multiplicative Decrease (LDMD) policy).

A discrete time network model that consists of $n$ users (flows) is considered ; Let $\{\ldots, t-1, t, t+$ $1, \ldots\}$ denote the discrete time instants which are assumed to coincide with the times at which all users receive feedback from their peer entities (synchronized feedback assumption). That is, the following assumption is made:

Assumption 1. (Non-delayed, synchronized feedback). All sources are assumed to receive the feedback instantaneously (zero propagation delay) and, thus, also simultaneously.

Let $f_{i}(t)$ denote the feedback received by user $i$ at time instant $t ; f_{i}(t)$ is not binary, but is of continuous value that specifies the packet loss rate that the corresponding flow $i$ experienced over the preceding time interval $(t-1, t)$. The following assumption is made regarding the induced packet losses.
Assumption 2. (Proportionality of losses) ${ }^{2}$. The total losses are assumed to be distributed to all flows in proportion to their rate. Consequently, the resulting loss rates will be identical, that is, $f_{i}(t)=f(t) \forall i$, and either all flows will experience packet losses or none.

The above assumption imply - as determined by the proposed scheme described below - that all flows will react in the same manner: they will all either increase (under zero losses) or decrease (under non-zero losses) their rate.

Let $x_{i}(t)$ denote the transmission rate of user $i$ over the interval $(t-1, t)$. The proposed DWAI/LDMD scheme is determined by the following function describing the next rate $\left(x_{i}(t+1)\right)$ in terms of the current $\left(x_{i}(t)\right)$ and the reported packet losses $f_{i}(t)$ :

$x_{i}(t+1)=$

$=\left\{\begin{array}{l}\min \left\{M, x_{i}(t)+\frac{M-x_{i}(t)}{M-m} I_{\mathrm{DW}}\right\} \text { if } f_{i}(t)=0 ; \\ \max \left\{m, x_{i}(t) d\left(1-f_{i}(t)\right)\right\} \text { if } f_{i}(t)>0 ;\end{array}\right.$

where, $M$ and $m$ are the maximum and minimum allowable rates $(M>m), I_{\mathrm{DW}}$ is the base increase step $\left(M-m>I_{\mathrm{DW}}>0\right)$, and $d$ is a constant which is less than one $(d<1)$. Notice that the normalized distance between the current rate $\left(x_{i}(t)\right)$ and the maximum allowable rate $M$ is taken into consideration, when the rate is increased; the normalization quantity is equal to the distance between the maximum and the minimum allowable rates. The increment to the rate is maximized (equal to $I_{\mathrm{DW}}$ ) when the rate is equal to the minimum allowable $(m)$ or the maximum allowable $M$ is very large, while the increment to the rate is minimum (equal to 0 ) when the rate is equal to the maximum allowable $(M)$. For a rate between the minimum and the maximum, the increment is a linear function of the distance of the current rate from the maximum allowable (equal to $I_{\mathrm{DW}} \frac{M-x_{i}(t)}{M-m}$ ). Although other (non-linear) functions of the distance could be considered to emphasize more or less large or small distances (for instance, $\left.\left(M-x_{i}(t)\right)^{2}\right)$ instead of $\left(M-x_{i}(t)\right)$, e.t.c.), this paper considers the linear

\footnotetext{
${ }^{2}$ For example, this would be a good approximation of FIFO queuing with small buffer size and packets whose size is small compared to the capacity of the link [25]. Because of the small packet size and the high link capacity a fluid model may accurately describe the behavior of the buffer. In view of this and since the buffer capacity is small proportional to rates losses are expected.
} 
function case only in an attempt to focus on bringing out the intrinsic advantages of considering the distance in the increment in a rigorous and mathematically tractable manner. Future investigation could focus on the consideration of other functions of the distance. Regarding the decrement to the rate when packet losses are reported, notice that the associated decrease factor $\left(d\left(1-f_{i}(t)\right)\right.$ is not constant - as in the case of the basic AI/MD scheme - but depends on the reported packet loss rate $f_{i}(t),\left(0<f_{i}(t) \leq 1\right)$.

Let $\vec{x}(t)=\left\{x_{1}(t), x_{2}(t), \ldots, x_{n}(t)\right\}$ denote the rate vector at time instant $t$ associated with $n$ supported users (flows) and let $X(t)$ denote the total network load associated to $\vec{x}(t)$. That is, $X(t)=$ $\sum_{i=1}^{n} x_{i}(t)$. Let $X_{\text {eff }}$ denote the targeted maximum total load which is the network capacity. Let $L(t)$ and $l_{i}(t)$ denote the number of packet losses for the network and user $i$, respectively, associated with the time instant $t$ (that is, occurred over $(t-1, t)$ ). Since the length of the interval $(t-1, t)$ is equal to 1 , $L(t)=1\left|X(t)-X_{\text {eff }}\right|^{+}$, where $|w|^{+}=w$ if $w>0$ and 0 otherwise. Let $f(t)$ and $f_{i}(t)$ denote the packet loss rate for the network and user (flow) $i$, respectively, associated with time instant $t$.

Since $f_{i}(t)=f(t), \forall i$ (see Assumption 2), $f_{i}(t)$ will be used instead of $f(t)$ only when the association with flow $i$ is to be emphasized. It should be noted that the interval $(t-1, t)$ is not of the time scale of round trip times but significantly larger. The $f_{i}(t)$ is reported by the feedback mechanism, e.g., RTCP Receiver Reports, therefore no packet loss estimation function is required, as it is required in the case when the feedbacks (acknowledgements) are in order of round trip times e.g., [4].

The study of the properties of the proposed DWAI/LDMD scheme (presented in the next sections) is facilitated by re-writing the rate adaptation equation (1) as follows

$$
\begin{aligned}
& x_{i}(t+1)= \\
& = \begin{cases}\min \left\{M, a_{\mathrm{I}}+b_{\mathrm{I}} x_{i}(t)\right\} & \text { if } f_{i}(t)=0 ; \\
\max \left\{m, a_{\mathrm{D}}+B_{\mathrm{D}}(f(t)) x_{i}(t)\right\} & \text { if } f_{i}(t)>0\end{cases}
\end{aligned}
$$

where

$$
\begin{aligned}
& a_{\mathrm{I}}=c M, \quad c=\frac{I_{\mathrm{DW}}}{M-m}, \quad 0<c<1 ; \\
& b_{\mathrm{I}}=1-c, \quad b_{\mathrm{I}}<1 \text {; } \\
& a_{\mathrm{D}}=0 \\
& B_{\mathrm{D}}(f(t))=d(1-f(t)), \quad B_{\mathrm{D}}(f(t))<1 ;
\end{aligned}
$$

For comparison purposes (to be used occasionally in the next sections), the rate adaptation equation for the basic $\mathrm{AI} / \mathrm{MD}$ scheme is given by

$$
\begin{aligned}
& x_{i}(t+1)= \\
& = \begin{cases}\min \left\{M, I_{\mathrm{AI}}+x_{i}(t)\right\} & \text { if } f(t)=0 ; \\
\max \left\{m, b_{\mathrm{D}} x_{i}(t)\right\} & \text { if } f(t)>0 ;\end{cases}
\end{aligned}
$$

where $I_{\mathrm{AI}}>0$ and $0<b_{\mathrm{D}}<1$. The size of $I_{\mathrm{AI}}$ and $I_{\mathrm{DW}}$ are considered to be not small but be of the order of the mean rate of a single video frame, that is, the rate increment corresponds to the increase of the frame rate by one.

Notice that the decrease factor $B_{\mathrm{D}}(f(t))$ under the LDMD policy is shaped by the induced packet losses, unlike the factor $b_{\mathrm{D}}$ under the basic MD policy. Thus, the LDMD policy applies a small decrease factor when losses are high and a large decrease factor when losses are low. The small decrease factor should help recover from congestion and reduce the losses quickly whereas the large decrease factor preserves the smoothness of the rate adaptation. Also notice that the rate increment $I_{\mathrm{DW}} \frac{M-x_{i}(t)}{M-m}$ under the DWAI policy is variable and is always equal to or less than the rate increment $I_{\mathrm{AI}}$ under the basic AI policy (assuming that $\left.I_{\mathrm{DW}}=I_{\mathrm{AI}}\right)^{3}$. Thus, the total and per flow rate increment under the DWAI policy will be lower than those under the basic AI policy, therefore the rate adaptation will be smoother under the DWAI policy. Also, the resulting load allocation among the flows will be fairer since the rate increment for a lower rate flow will be larger than that of a higher rate flow. These claims are also supported by the numerical results. The improved smoothness and convergence to fairness properties of the DWAI/LDMD scheme compared to those properties under the AI/MD scheme make it particularly tailored to controlling the rates of $\mathrm{CM}$ applications.

The minimum rate $m$ corresponds to the lower acceptable perceptual quality for a flow. The scheme does not allow for rates below this lower bound. Note that, we consider that $m$ is static and common for all flows in the same class. A typical range of values for $m$ could be $34-56 \mathrm{~kb} / \mathrm{s}$, which are the minimum rates for webcasting of $\mathrm{CM}$ flows at an acceptable quality. For data transfer services, $m$ could be equal to 0 . The

\footnotetext{
${ }^{3} I_{\mathrm{DW}}$ is selected to be equal to $I_{\mathrm{AI}}$, so that the rate increment $I_{\mathrm{DW}} \frac{M-x_{i}(t)}{M-m} \leq I_{\mathrm{AI}}$.
} 
maximum rate $M$ corresponds to a specific level of perceptual quality. This level could be: (a) a high level of perceptual quality, over which the gain of the perceived quality is relatively low, e.g., $4.5 \mathrm{Mb} / \mathrm{s}$ for MPEG-4 flows in a potential range of $2.5-6 \mathrm{Mb} / \mathrm{s}$; (b) the limit of the video encoder or encoding scheme, e.g., $1.5 \mathrm{Mb} / \mathrm{s}$ for $\mathrm{H} .263$ video flows in a potential range of $0.768-2 \mathrm{Mb} / \mathrm{s}$; (c) the maximum transmission rate, e.g., $3 \mathrm{Mb} / \mathrm{s}$ for ADSL users in a potential range of $1-6 \mathrm{Mb} / \mathrm{s}$. $\mathrm{M}$ has to be selected properly to match the requirements of a specific class and not selected so that to match the capacity of the network. The value of $\mathrm{M}$ cannot be arbitrarily very large but is subjected to the constraints of the video encoders and supported encoding scheme or to a maximum transmission rate.

In the context of this paper we consider that the maximum rate $M$ is static - in the sence that $M$ is predetemined for each class and cannot be updated and common for all flows in the same class. Flows with different $M$ is assumed to be isolated. That is, MPEG-4 flows with $M=4 \mathrm{Mb} / \mathrm{s}, \mathrm{H} .263$ flows with $M=2 \mathrm{Mb} / \mathrm{s}$ or HDTV flows is assumed to belong in different classes. An application entity may explicitly informs each source about the available classes and the corresponding values of $M$. The source is free to select the preferable class among the set of potential classes, according the sources's QoS requirements.

However, in some cases where $M$ can take values in a wide range, $M$ might be considered to be configurable, in the sence that $M$ can be updated, in order to exploit future bandwidth upgrades. In case of a link bandwidth upgrade ( $X_{\text {eff }}$ is increased), the administrator may or may not increase $M$. In the former case the upper bound of the perceived quality that a flow could obtain is increased, whereas in the latter case, the number of flows that could obtain the initial upper bound $M$ is increased. An application entity may $e x-$ plicitly determine the upper bound $M$ for each class. This entity is aware of the values of $X_{\mathrm{eff}}, M$ (set by an administrator). When a source initiates a flow, it communicates to the application entity which returns the value of $M$ for the specific class. All sources are updated by the entity for the current value of $M$ when the latter is adjusted, so that the value of $M$ be common to all sources. An example of an application entity that explicitly controls the rate of $\mathrm{CM}$ applications in the current best-effort Internet is the H.323 Gate- keeper ${ }^{4}$ [31].

An alternative way to the explicit determination of $M$ is the dynamic determination. The sources may dynamically determine the upper bound $\mathrm{M}$ as a function of the fair share, provided that each source can estimate the fair share, based on locally available information such as the packet loss rate and the values of the control parameters. Flows that share a common network will estimate common fair share and $M$. In this case, $M$ may dynamically be adjusted in order to match the current fair share, e.g., be twice the fair share. Thus, under bandwidth availability (number of flows is decreased or bandwidth links is upgraded) $M$ is increased, otherwise it is decreased. When $M$ is decreased the rate increment $\frac{M-x_{i}(t)}{M-m} I_{\mathrm{DW}}$ is decreased as well, resulting in less aggressive increments and smoother adaptation behavior. The dynamic determination of $M$ by the sources, without the need of any application entity, is closer to the decentralized philosophy of the Internet and Differentiated Services. The fair share estimation is outside the scope of this paper, however in Section 4.3 a hint is given on how the proposed scheme can compute an estimation of the fair share.

\section{Convergence to Efficiency and Fairness}

In this section it is shown that the proposed DWAI/LDMD scheme (a) converges to the efficiency line $X_{\text {eff }}$ provided that $X_{\text {eff }}<n M$, and (b) converges to fairness.

\subsection{Convergence to Efficiency}

Let $X_{\text {eff }}$ represent a targeted total load which may typically be the network capacity. It is assumed that $n m<X_{\text {eff }}<n M$, otherwise either the network will be always congested (if $n m>X_{\text {eff }}$ ) or the network's load will never reach the efficiency line (if $X_{\text {eff }}>$ $n M)$.

Proposition 1. The DWAI/LDMD scheme converges to efficiency.

Proof. As it is discussed in more detail in [3], the efficiency line is reached as long as the total rate increases (decreases) when the common feedback to all

\footnotetext{
${ }^{4}$ One of the control capabilities of an H.323 Gatekeeper is to control the upper rate of videoconferencing sessions so that the bandwidth be not seized by H.323 videoconferencing applications.
} 
users dictates a rate increase (decrease). That is,

$$
\begin{aligned}
& f_{i}(t)=0 \Rightarrow \sum_{i=1}^{n} x_{i}(t+1) \geq \sum_{i=1}^{n} x_{i}(t) \\
& f_{i}(t)>0 \Rightarrow \sum_{i=1}^{n} x_{i}(t+1)<\sum_{i=1}^{n} x_{i}(t)
\end{aligned}
$$

Indeed the inequalities in (6) are always true, as it may be logically concluded.

\subsection{Discussion on Fairness Properties}

The fairness index $F(\vec{x}(t))$ associated with a rate vector $\vec{x}(t)$, where $F(\vec{x}(t))=\frac{\left(\sum_{i=1}^{n} x_{i}(t)\right)^{2}}{n \sum_{i=1}^{n} x_{i}(t)^{2}}$, has been used widely in the past, [3,18], to investigate whether a scheme converges to the fairness line after any sequence of increase and decrease steps. A scheme converges to fairness if $\lim _{t \rightarrow \infty} F(\vec{x}(t))=1$, which means that for large $t, x_{1}(t) \approx x_{2}(t) \approx \ldots \approx$ $x_{n}(t) \approx \frac{X_{\text {eff }}}{n}$. The value of $F(\vec{x}(t))$ is always less or equal to 1 [3].

A new index which captures the evolution of fairness in a manner closer to the physical meaning of fairness than the fairness index $F(\vec{x}(t))$ is introduced. The fairness index $F(\vec{x}(t))$ measures the "relative" distance from fairness. Therefore, if the user allocation is proportionally reduced for all the users - as is the case with the proposed DWAI/LDMD scheme since $a_{\mathrm{D}}=0$ (see (4)) - the value of the index will remain the same. In Fig. 1 a two-dimensional rate vector $\vec{x}(t)(\equiv \overrightarrow{A B})$ is depicted. When the rate is proportionally decreased $\vec{x}(t+1) \equiv \overrightarrow{A Z}$ will remain along the line $\mathrm{AB}$ and will be smaller than $\vec{x}(t)$. Although the distance from the fairness line $x_{j}(t)=x_{i}(t)$ is reduced ( $\mathrm{ZH}$ versus $\mathrm{BC}$ ), the fairness index $F(t)$ - as shown earlier - will remain the same. This fact shows that the fairness index does not capture a fairness improvement in absolute terms.

In the sequel, the new index $D F_{i, j}(t)$ is defined, representing the distance (unfairness) of point $\left(x_{i}(t), x_{j}(t)\right)$ from the fairness line. This index is used to derive an alternative proof of the convergence of the proposed scheme to fairness that allows for better monitoring of the fairness evolution. Fig. 1 depicts the index $D F_{i, j}(t)$ (三 BC) associated with rates $x_{i}(t)$ and $x_{j}(t)$ (point $\mathrm{B}$ ) at time $t$, assuming that $x_{j}(t)>x_{i}(t)$. The evolution of this index captures the fairness improvement after an adaptation step. From Fig. 1 it is easily derived that the Euclidean distance $B C$ of the point $\left(x_{i}(t), x_{j}(t)\right)$ from the fairness line is equal to $\frac{B D}{2}$. Since $B D^{2}=$

$$
\begin{aligned}
& B E^{2}+E D^{2}=2\left|x_{j}(t)-x_{i}(t)\right|^{2} \text { it is derived that } \\
& B C=D F_{i, j}(t)=\frac{\left|x_{j}(t)-x_{i}(t)\right|}{\sqrt{2}} \geq 0 .
\end{aligned}
$$

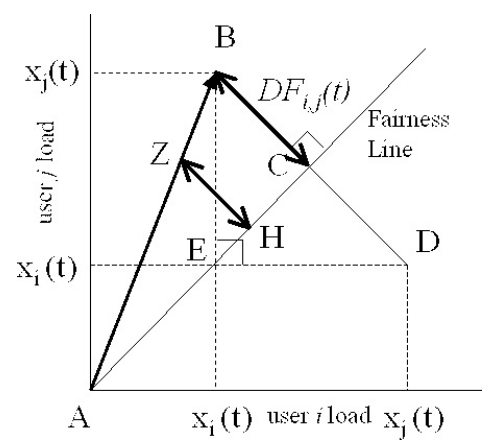

Figure 1. The distance $D F_{i, j}(t)$ of point $\left(x_{i}(t), x_{j}(t)\right)$ from the fairness line.

The new index $D F_{i, j}(t)$ allows for simpler derivations and more elegance proofs than the index $F(\vec{x}(t))$. In addition, it captures the convergence speed to fairness - see $D F_{i, j}(t)$ in Corrolary 2 that follows - and therefore the simple comparison of the convergence speeds to fairness of different schemes, which is not that simple by exploiting index $F(\vec{x}(t))$. However, index $F(\vec{x}(t))$ is used for depicting the convergence speed to fairness in the figures, since this index is very common in the research literature.

The following proposition shows that under both the proposed DWAI and LDMD policies the (absolute) distance from the fairness line is reduced.

Proposition 2. Fairness is improved after a load increase (decrease) action under the DWAI (LDMD) policy.

Proof. Consider any pair of loads $\left(x_{i}(t), x_{j}(t)\right)$ associated with the load vector $\vec{x}(t)$ and, without loss of generality, assume that $x_{j}(t)>x_{i}(t)$. The index $D F_{i, j}^{+}(t+1)$ at the next time instant $t+1$ after a load increase action is expressed in terms of the index $D F_{i, j}(t)=\frac{\left|x_{j}(t)-x_{i}(t)\right|}{\sqrt{2}}$ at time instant $t$ as follows.

$D F_{i, j}^{+}(t+1)=\frac{\left|x_{j}(t+1)-x_{i}(t+1)\right|}{\sqrt{2}}$ 


$$
\begin{aligned}
& =\frac{(1-c)\left|x_{j}(t)-x_{i}(t)\right|}{\sqrt{2}} \\
& =(1-c) D F_{i, j}(t)
\end{aligned}
$$

Since $(1-c)<1$, it is concluded that $D F_{i, j}^{+}(t+1)<$ $D F_{i, j}(t)$. That is, the (Euclidean) distance from the fairness line is decreased after a load increase action. The index $D F_{i, j}^{-}(t+1)$ at time instant $t+1$, following load decrease action, is expressed in terms of $D F_{i, j}(t)$ as follows.

$$
\begin{aligned}
D F_{i, j}^{-}(t+1) & =\frac{\left|x_{j}(t+1)-x_{i}(t+1)\right|}{\sqrt{2}} \\
& =d(1-f(t)) D F_{i, j}(t)
\end{aligned}
$$

Since $d(1-f(t))<1$, it is concluded that $D F_{i, j}^{-}(t+$ $1)<D F_{i, j}(t)$. That is the distance from the fairness line is decreased after a load decrease action. Since the fairness associated with any pair $\left(x_{i}(t), x_{j}(t)\right)$ is improved following any action (load increase or decrease), the fairness for all flows is improved.

The following proposition shows that under the basic AI policy the distance from the fairness line remains the same, whereas under the basic MD policy the distance is reduced. By referring to Fig. 1 and from (5) the proof is easily derived.

Proposition 3. Fairness is not improved after a load increase action under the basic AI policy. It is improved after a load decrease action under the basic MD policy.

Also, the following Corollary is self-evident.

Corollary 1. The LDMD policy presents better fairness improvement after a load rate decrease action than the basic MD policy if $d(1-f(t))<b_{\mathrm{D}}$; this holds true if $d=b_{\mathrm{D}}$.

It should be noted that the fairness improvement under the DWAI policy with respect to the absolute fairness metric $D F_{i, j}(t)$ may be attributed to the $d i$ verse rate increment for the two (unequal rate) flows. This is not the case under the basic AI policy and thus fairness is not improved with respect to this fairness metric. In view of the earlier discussion the following Corollary that shows the fairness improvement under the DWAI/LDMD scheme, is easily derived.
Corollary 2. The distance from the fairness line when the $z^{\text {th }}$ decrease step occurs, under the DWAI/LDMD scheme, is given by (8), and it vanishes as $z \rightarrow \infty$.

$D F_{i, j}\left(t_{z}\right)=(1-c)^{k} d^{z} \prod_{h=1}^{z}\left(1-f\left(t_{h}\right)\right) D F_{i, j}(t)$

where $k$ denotes the total number of increase steps and $t_{h}$ refers to the time instant of the $h^{\text {th }}$ decrease step.

Proof. Let $k_{1}$ denote the number of required increase steps to reach the efficiency line. From the proof of Proposition 2, is easily derived that $D F_{i, j}\left(t+k_{1}\right)=$ $(1-c)^{k_{1}} D F_{i, j}(t)$. After the subsequent decrease action (under the LDMD policy) at time instant $t_{1}$ the associated index, $D F_{i, j}\left(t_{1}\right)$, is given by $D F_{i, j}\left(t_{1}\right)=$ $\left(1-f\left(t_{1}\right)\right)(1-c)^{k_{1}} D F_{i, j}(t)$. Let $z$ denote the number of decrease steps and $k$ the total number of increase steps. The index $D F_{i, j}\left(t_{z}\right)$ after the $z^{\text {th }}$ decrease step is then easily shown to be given by (8). From equation (8) it is concluded that $D F_{i, j}\left(t_{z}\right) \rightarrow 0$ as $z \rightarrow \infty$, since $\left(1-f\left(t_{h}\right)\right)<1, \forall h \leq z$ and $(1-c)<1$. Thus, the convergence to fairness has been proved in terms of the index $D F_{i, j}(t)$, as well.

\section{Analysis of the Behavior of the DWAI/LDMD Scheme}

\subsection{Responsiveness to BW availability of the DWAI policy}

In the sequel the responsiveness to BW availability (convergence time to efficiency) under the DWAI policy is quantified by determining the number $k_{\mathrm{DW}}$ of successive rate increases (steps) required in order for the network to reach the efficiency line $X_{\text {eff }}$, from an initial total load $X(t)$. BW may become available due to (a) initial startup, (b) flow termination(s), and (c) rate decrease actions after congestion detection.

Proposition 4. Under the DWAI policy, the rate $x_{i}(t+k)$ of flow $i$ after $k$ successive increase steps, starting from some initial rate $x_{i}(t) \in(m, M)$, satisfies the following:

(a) $\begin{aligned} & x_{i}(t+k)=x_{i}(t)(1-c)^{k}+M\left(1-(1-c)^{k}\right) \\ & =M-\left(M-x_{i}(t)\right)(1-c)^{k}\end{aligned}$

(b) $x_{i}(t+k)<M$ for any $k \geq 1$ 
Proof. The proof is based on mathematical induction. In $l=1$ increase steps (9) clearly holds since from (2) and (3) it is derived that $x_{i}(t+1)=x_{i}(t)(1-c)+$ $M(1-1+c)=x_{i}(t)(1-c)+M c$. Assume now that (9) is true for $k=l$ as well. That is, $x_{i}(t+l)=$ $x_{i}(t)(1-c)^{l}+M\left(1-(1-c)^{l}\right)$. Then,

$$
\begin{aligned}
& x_{i}(t+l+1)=x_{i}(t+l)(1-c)+c M \\
& =\left(x_{i}(t)(1-c)^{l}+M\left(1-(1-c)^{l}\right)\right)(1-c)+c M \\
& =x_{i}(t)(1-c)^{l+1}+M\left(1-(1-c)^{l+1}\right)
\end{aligned}
$$

and, thus, (9) is shown to hold for $k=l+1$ as well, which concludes the proof of part (a). Since $x_{i}(t)<$ $M$, it is derived from (9) that $x_{i}(t+k)<M$ for any $k \geq 1$.

The following corollary is self-evident in view of Proposition 4.

Corollary 3. Given an initial n-dimensional rate vector $\vec{x}(t)$ with total network load $X(t)$, the total network load $X(t+k)$, after $k$ successive increases is given by

$$
\begin{aligned}
X(t+k) & =(1-c)^{k} X(t)+n M\left(1-(1-c)^{k}\right) \\
& =n M-(n M-X(t))(1-c)^{k}
\end{aligned}
$$

Let $k_{\mathrm{DW}}(X(t))$ denote the number of successive increase steps required in order for the network load to reach $X_{\text {eff }}$ starting from $X(t) . k_{\mathrm{DW}}(X(t))$ is given by the expression:

$$
\begin{aligned}
& X_{\mathrm{eff}}=(1-c)^{k} X(t)+n M\left(1-(1-c)^{k}\right) \Leftrightarrow \\
& (1-c)^{k}=\frac{n M-X_{\mathrm{eff}}}{n M-X(t)} \Rightarrow \\
& k_{\mathrm{DW}}(X(t))=\left[\log _{(1-c)} \frac{n M-X_{\mathrm{eff}}}{n M-X(t)}\right]
\end{aligned}
$$

where $\lceil w\rceil$ is the smallest integer exceeding $w$. $k_{\mathrm{DW}}(X(t))$ determines the responsiveness to $\mathrm{BW}$ availability and is independent of the initial load vector $\vec{x}(t)$.

\subsection{Periodic oscillatory behavior of the DWAI/LDMD scheme}

In this section it is established that following the first application of the LDMD policy (that occurs when the total load exceeds $X_{\text {eff }}$ for the first time and packet losses are reported) the rate adaptation behavior is periodic. That is, the total load fluctuates between fixed levels below and above the efficiency line in a periodic fashion.

Let $t_{j}$ denote the $j^{t h}$ overload time instant, that is the $j^{\text {th }}$ time in which the total load $X(t)$ exceeds $X_{\text {eff }}$ (that is, $\left.X\left(t_{j}\right)>X_{\text {eff }}\right), j \geq 1$. The following proposition determines the behavior of the total load in the next time instant $X\left(t_{j}+1\right)$ and the resulting oscillation below the efficiency line, $S^{-}\left(t_{j}+1\right)$, defined below.

Proposition 5. If $t_{j}$ is the $j^{\text {th }}$ overload time instant then:

(a) $X\left(t_{j}+1\right)=d X_{\text {eff }}$ and, thus, the total load in the next time instant always falls below the efficiency line and this new total load is always the same, independent of $X\left(t_{j}\right)$.

(b) $S^{-}\left(t_{j}+1\right)=X_{\text {eff }}-X\left(t_{j}+1\right)=(1-d) X_{\text {eff }}$ which is independent of $X\left(t_{j}\right)$ and, thus, the size of the oscillations below the efficiency line caused by decrease actions are constant.

Proof. Since $X\left(t_{j}\right)>X_{\text {eff }}$ and $f_{i}\left(t_{j}\right)=f\left(t_{j}\right)>$ 0 , the total load will be decreased according to the LDMD policy at time instant $t_{j}+1$. The new total rate at $t_{j}+1$ is given by (see Assumption 2):

$$
\begin{aligned}
& X\left(t_{j}+1\right)=\sum_{i=1}^{n} x_{i}\left(t_{j}+1\right)=\sum_{i=1}^{n} d\left(1-f_{i}\left(t_{j}\right)\right) x_{i}\left(t_{j}\right) \\
& =d\left(1-\frac{X\left(t_{j}\right)-X_{\mathrm{eff}}}{X\left(t_{j}\right)}\right) X\left(t_{j}\right)=d X_{\mathrm{eff}}
\end{aligned}
$$

which proves (a) and (b).

It is obvious that the value of $d$ should be less that 1 to ensure that the total load falls below the efficiency line.

After a decrease step, the total load $X(t)$ will start increasing from an initial load $d X_{\text {eff }}$ until it exceeds the efficiency line; let $k^{\mathrm{T}}$ denote the required number of increase steps. $k^{\mathrm{T}}$ is a metric of the responsiveness to small BW availability (due to a rate decrease action(s) as a reaction to congestion) in the sense that captures the required number of intervals (increase steps) to reach the efficiency line after such a rate decrease. In view of Proposition 5, after $k^{\mathrm{T}}$ increase steps the total load $X(t)$ will be reduced again 
to $d X_{\text {eff }}$ in the next time instant and thus the total load adaptation behavior will be periodic: the total load will be at the overload level $X\left(t_{j}\right)$ at time instant $t_{j}$, $j>1$, then will fall at the level $d X_{\text {eff }}$ in the next time instant and then climb up to the same level $X\left(t_{j}\right)$ after $k^{\mathrm{T}}$ steps always in the same manner (reaching time instant $t_{j+1}=t_{j}+1+k^{\mathrm{T}}$ from which the process will be repeated). Thus, the function of the total load $X(t), t>t_{1}$ is periodic with period $T=k^{\mathrm{T}}+1$. It should be noted that $X\left(t_{j}\right), j>1$, is independent of the initial total load $X(0)$ and fixed. The size of the oscillations above $X_{\text {eff }}, S^{+}$, below $X_{\text {eff }}, S^{-}$, and the induced packet loss rates $f^{\mathrm{c}}$ (over a period $T$ ) are given by:

$$
\begin{aligned}
S^{+} & =X\left(t_{j}\right)-X_{\mathrm{eff}}, j>1 \text { fixed for all } j>1(11) \\
f^{\mathrm{c}} & =\frac{S^{+}}{X\left(t_{j}\right)}=\frac{S^{+}}{X_{\mathrm{eff}}+S^{+}}=1-\frac{X_{\mathrm{eff}}}{X\left(t_{j}\right)} \\
S^{-} & =(1-d) X_{\mathrm{eff}}
\end{aligned}
$$

Regarding $X\left(t_{1}\right)$, that is the total load when the efficiency line is exceeded for the first time, it should be noted that it will - in general - be different than $X\left(t_{j}\right), j>1$, since it would be determined by a sequence of increase steps starting from some initial value $X(0)$ and not $d X_{\text {eff }}$ and therefore would depend on $X(0)$. Fig 2 shows a typical sample path of the total load. Notice that $X\left(t_{1}\right) \neq X\left(t_{j}\right), j>1$, while all rate decrease actions bring the load to the same level $d X_{\text {eff }}$.

Since $f^{c}$ is fixed due to the periodic oscillatory behavior discussed above, it is concluded that the decrease factor $B_{\mathrm{D}}(f(t))$ will also have a fixed value as long as the number $n$ of the flows remains unchanged (and, thus, $f^{\mathrm{c}}$ remains the same).

\subsection{Size of Oscillations and Packet Loss}

In view of the earlier discussion, after a decrease step the total load $X(t)$ starts increasing from load $d X_{\text {eff }}$ (see Proposition 5 and Fig. 2). The total load $X(t)$ will exceed the efficiency line after $k_{\mathrm{DW}}^{\mathrm{T}}$ increase steps, where $k_{\mathrm{DW}}^{\mathrm{T}}$ is given by (10) for $X(t)=$ $d X_{\text {eff }}$ :

$k_{\mathrm{DW}}^{\mathrm{T}}=\left\lceil\log _{(1-c)} \frac{n M-X_{\mathrm{eff}}}{n M-d X_{\mathrm{eff}}}\right\rceil$

Since the base $(1-c)$ decreases (increases) as $I_{\mathrm{DW}}$ increases (decreases) (see $(3)$, recall that $1-c<1$ )

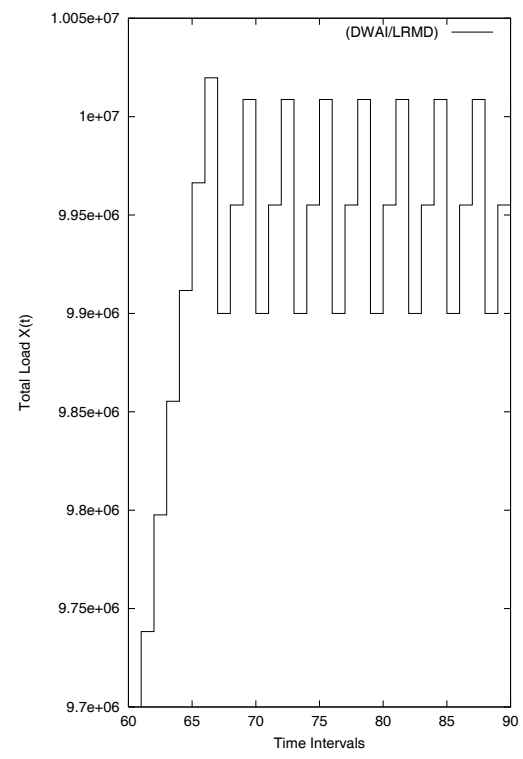

Figure 2. Adaptation behavior of the DWAI/LDMD scheme with $d=0.99, n=10, m=56 \mathrm{~kb} / \mathrm{s}, M=$ $1.2 \mathrm{Mb} / \mathrm{s}, I_{\mathrm{DW}}=30 \mathrm{~kb} / \mathrm{s}, X_{\mathrm{eff}}=10 \mathrm{Mb} / \mathrm{s}$.

it is concluded that $k_{\mathrm{DW}}^{\mathrm{T}}$ decreases (increases) with $I_{\mathrm{DW}}$, as expected.

Clearly, $k_{\mathrm{DW}}^{\mathrm{T}}$ decreases with $d, 0<d<1$ and $k_{\mathrm{DW}}^{\mathrm{T}} \rightarrow 1$ as $d \rightarrow 1$. Thus, $d$ affects the number of increase steps required to reach the efficiency line from $d X_{\text {eff. }}$. For a value sufficiently close to 1 , it will require only one increase step to exceed the efficiency line. For a given $d$, a threshold $n_{e}(d)$ may be determined such that $k_{\mathrm{DW}}^{\mathrm{T}}=1$ as long as the number of flows $n$ exceeds $n_{e}(d)$. For $d=$ $0.99, M=1.2 \mathrm{Mb} / \mathrm{s}, X_{\text {eff }}=10 \mathrm{Mb} / \mathrm{s}$ it turns out that $n_{e}(0.99)=12$. The total load $X\left(t_{j}\right), j>1$ when the efficiency line is exceeded again is determined by the DWAI policy and it is given by (see Corollary 3):

$X\left(t_{j}\right)=n M-\left(n M-d X_{\mathrm{eff}}\right)(1-c)^{k_{\mathrm{DW}}^{\mathrm{T}}}, j>1$

In view of (15), $S^{+}$and $f^{c}$ (see (11) and (12)) become:

$S^{+}=n M-\left(n M-d X_{\mathrm{eff}}\right)(1-c)^{k_{\mathrm{DW}}^{\mathrm{T}}}-X_{\mathrm{eff}}$ 


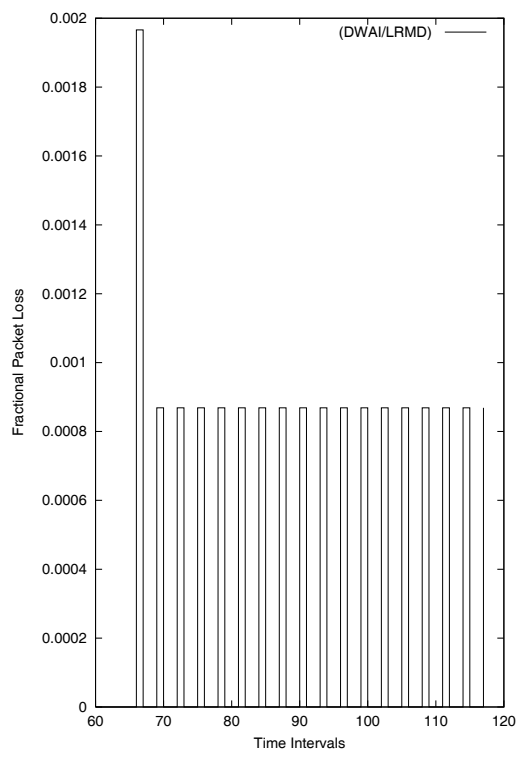

Figure 3. Packet loss rate under the DWAI/LDMD scheme with $d=0.99, b_{\mathrm{D}}=0.9, n=10, m=$ $56 \mathrm{~kb} / \mathrm{s}, M=1.2 \mathrm{Mb} / \mathrm{s}, I_{\mathrm{DW}}=30 \mathrm{~kb} / \mathrm{s}, X_{\mathrm{eff}}=$ $10 \mathrm{Mb} / \mathrm{s}$.

$f^{c}=1-\frac{X_{\mathrm{eff}}}{n M-\left(n M-d X_{\mathrm{eff}}\right)(1-c)^{k_{\mathrm{DW}}^{\mathrm{T}}}}$

Regarding $X\left(t_{1}\right)$, that it is - in general - different than (15) it is obtained from Corollary 3 that:

$X\left(t_{1}\right)=n M-(n M-X(0))(1-c)^{k_{\mathrm{DW}}(X(0))}$

Fig. 3 illustrates the induced packet loss rate under the DWAI/LDMD scheme. The plots shown in Fig. 2 and 3 are generated from Matlab simulations. The above may be summarized in the following proposition.

Proposition 6. The DWAI/LDMD scheme presents a regular (periodic) oscillatory adaptation behavior. After the first overload time instant $t_{1}$ the total load $X(t)$ exceeds the efficiency line every $k_{\mathrm{DW}}^{\mathrm{T}}+1$ time instants, where $k_{\mathrm{DW}}^{\mathrm{T}}$ is given by (14). Following $t_{1}$, the overshoot $S^{+}$, the undershoot $S^{-}$and the packet loss rate $f^{\mathrm{c}}$ are fixed, and given by (16), (13) and (17), respectively.

Solving equation (17) for $\frac{X_{\text {eff }}}{n}$, the fair share can be derived. This value can be used to determine $M$ in the case of the implicit determination of $M$, as discussed in the last paragraph of Section 2.

\subsection{Throughput}

Since the behavior of the total load $X(t)$ is periodic (after a short initial transient period and for unchanged flow composition), the mean throughput when $t \rightarrow \infty$ will be equal to the mean throughput as established over a period. The total load successfully transmitted during a period, $L_{\mathrm{T}}$, is equal to the sum of $X\left(t_{j-1}+1\right) \cdots X\left(t_{j-1}+k_{\mathrm{DW}}^{\mathrm{T}}+1\right)\left(=X\left(t_{j}\right)\right)$, which is the total load transmitted during a period, minus the load failed to be transmitted during the time interval $\left[t_{j}-1, t_{j}\right]$, which is equal to $f\left(t_{j}\right) X\left(t_{j}\right)$, and given by:

$$
\begin{aligned}
L^{\mathrm{T}}= & \sum_{i=0}^{k_{\mathrm{DW}}^{\mathrm{T}}} X\left(t_{j-1}+1+i\right)-f\left(t_{j}\right) X\left(t_{j}\right) \\
= & \sum_{i=0}^{k_{\mathrm{DW}}^{\mathrm{T}}}\left(n M-\left(n M-d X_{\mathrm{eff}}\right)(1-c)^{i}\right) \\
= & -n M+\left(n M-d X_{\mathrm{eff}}\right)(1-c)^{k_{\mathrm{DW}}^{\mathrm{T}}}+X_{\mathrm{eff}} \\
& -\left(n M-d X_{\mathrm{eff}}\right) \frac{1-c(1-c)^{k_{\mathrm{DW}}^{\mathrm{T}}}}{c}
\end{aligned}
$$

Thus, the mean throughput, $\overline{R^{\mathrm{T}}}$, under the DWAI/LDMD scheme is the fraction of $L^{\mathrm{T}}$ to the maximum possible successfully transmitted load over a period, that is $X_{\text {eff }}$ multiplied by the period duration $k_{\mathrm{DW}}^{\mathrm{T}}+1$, and given by:

$$
\begin{aligned}
& \overline{R^{T}}=\frac{L^{\mathrm{T}}}{\left(k_{\mathrm{DW}}^{\mathrm{T}}+1\right) X_{\mathrm{eff}}}= \\
& =\frac{X_{\mathrm{eff}}+k_{\mathrm{DW}}^{\mathrm{T}} n M-\left(n M-d X_{\mathrm{eff}}\right) \frac{1-c(1-c)^{k_{\mathrm{DW}}^{\mathrm{T}}}}{c}}{\left(k_{\mathrm{DW}}^{\mathrm{T}}+1\right) X_{\mathrm{eff}}}
\end{aligned}
$$

Equation (18) may also be written as:

$$
\begin{aligned}
& \sum_{i=0}^{k_{\mathrm{DW}}^{\mathrm{T}}}\left(d X_{\mathrm{eff}}+D_{i}\right)-f\left(t_{j}\right) X\left(t_{j}\right)= \\
& \left(k_{\mathrm{DW}}^{\mathrm{T}}+1\right) d X_{\mathrm{eff}}+\sum_{i=0}^{k_{\mathrm{DW}}^{\mathrm{T}}} D_{i}-f\left(t_{j}\right) X\left(t_{j}\right)
\end{aligned}
$$$$
\text { where } D_{i}=X\left(t_{j-1}+1+i\right)-d X_{\text {eff }} \geq 0 \text {. }
$$ 
and $\overline{R^{\mathrm{T}}}$ could be written as

$$
\begin{aligned}
& \frac{\left(k_{\mathrm{DW}}^{\mathrm{T}}+1\right) d X_{\mathrm{eff}}+\sum_{i=0}^{k_{\mathrm{DW}}^{\mathrm{T}}} D_{i}-f\left(t_{j}\right) X\left(t_{j}\right)}{\left(k_{\mathrm{DW}}^{\mathrm{T}}+1\right) X_{\mathrm{eff}}}= \\
& d+\frac{\sum_{i=0}^{k_{\mathrm{DW}}^{\mathrm{T}}} D_{i}-f\left(t_{j}\right) X\left(t_{j}\right)}{\left(k_{\mathrm{DW}}^{\mathrm{T}}+1\right) X_{\mathrm{eff}}}
\end{aligned}
$$

It is obvious that the term $\sum_{i=0}^{k_{\mathrm{DW}}^{\mathrm{T}}} D_{i}-f\left(t_{j}\right) X\left(t_{j}\right)$ is greater than 0 , therefore, the throughput under the DWAI/LDMD scheme is always greater than the parameter $d$. Thus, $d$ could be considered as a lower bound on the throughput guaranteed by the DWAI/LDMD scheme. It should be noted that this lower bound is independent of $X(0)$, the number of flows $n$ and $X_{\text {eff. }}$ This is a nice property of the LDMD policy.

\section{Discussion on the Comparative Performance of the DWAI/LDMD and AI/MD schemes}

\subsection{Convergence Speed to Fairness}

In this section the convergence speed to fairness under the DWAI/LDMD scheme is discussed and compared against the corresponding under the basic $\mathrm{AI} / \mathrm{MD}$ scheme.

In view of Proposition 6, Corollary 2 could be stated as follows:

Corollary 4. The distance from the fairness line when the $p^{\text {th }}$ period is completed, under the DWAI/LDMD scheme, is given by:

$$
\begin{array}{r}
D F_{i, j_{\mathrm{DWLD}}}\left(t_{z}\right)=(1-c)^{k_{\mathrm{DW}}} d\left(1-f\left(t_{1}\right)\right) \times \\
\left((1-c)^{k_{\mathrm{DW}}^{\mathrm{T}}} d\left(1-f^{\mathrm{c}}\right)\right)^{p} D F_{i, j}(t)
\end{array}
$$

From Proposition 2 (see equation (7)) it may be noted that the value of the index $D F_{i, j_{\text {DWLD }}}(t+1)$ depends on the reported packet loss rate $f(t)$. This implies that a fast convergence to fairness is expected under the LDMD policy after the introduction of a new user in the system that causes high loss rates, which is desirable under such conditions. The former may not be the case under the basic MD policy that applies a fixed decrease factor independently of the induced packet loss. Also, it should be noticed that the convergence speed to fairness under the AI/MD scheme depends on the factor $b_{\mathrm{D}}$. The lower $b_{\mathrm{D}}$ the better the convergence speed to fairness and the worse

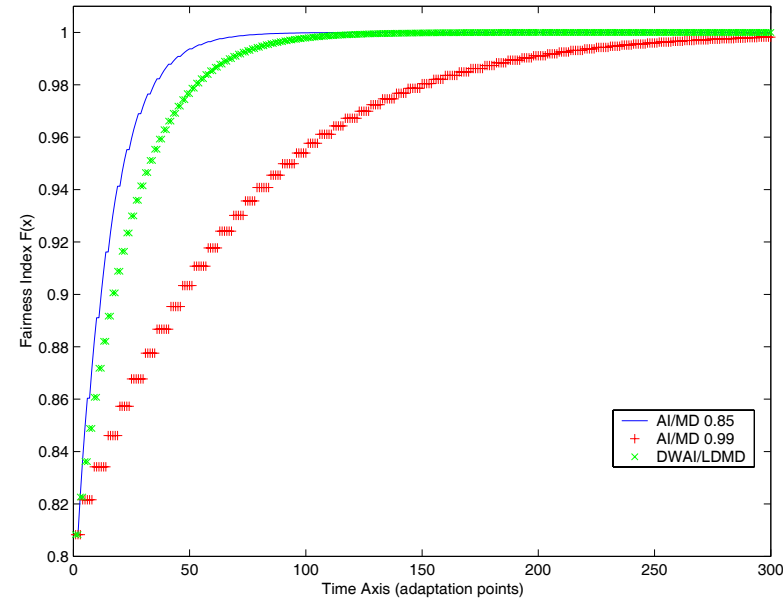

Figure 4. Fairness Index $F(\vec{x}(t))$ under the DWAI/LDMD and basic AI/MD schemes.

the smoothness. This is depicted in Fig. 4 which illustrates the evolution of the fairness index $F(\vec{x}(t))$ under the DWAI/LDMD scheme $\left(d=0.99, I_{\mathrm{DW}}=\right.$ $30 \mathrm{~kb} / \mathrm{s}$ ) and two applications of the basic AI/MD scheme with $I_{\mathrm{AI}}=30 \mathrm{~kb} / \mathrm{s}$ and $b_{\mathrm{D}}=0.99$ and $b_{\mathrm{D}}=$ 0.85 , respectively. The initial rate vector is given by $\vec{x}=\{m+1 *(M-m) / n, m+2(M-m) / n, \cdots, M\}$ where $m=56 \mathrm{~kb} / \mathrm{s}, M=1.2 \mathrm{Mb} / \mathrm{s}, n=12$ and, thus, the initial total load is $X(0)=8,108,000>$ $X_{\text {eff }}=8 \mathrm{Mb} / \mathrm{s}$. As expected, the curve representing the fairness index under the DWAI/LDMD scheme converges faster to 1 than the curve under the basic $\mathrm{AI} / \mathrm{MD}$ with $b_{\mathrm{D}}=0.99=d \approx B_{\mathrm{D}}(t)$ due to the fact that the DWAI policy converges faster than the basic AI policy. It should be noticed that the convergence speed to fairness under the basic AI/MD scheme is improved as the parameter $b_{\mathrm{D}}$ is decreased i.e., the convergence speed under the basic $\mathrm{AI} / \mathrm{MD}$ scheme with $b_{\mathrm{D}}=0.85$ is better than that under the DWAI/LDMD scheme (see the associated curve in Fig. 4).

\subsection{Convergence to Efficiency}

As discussed earlier in Section 3, the fairness is improved under the DWAI policy (Proposition 2) whereas remains the same under the basic AI policy (Proposition 3). However, the responsiveness to BW 
availability (convergence speed to efficiency) deteriorates since the rate increment under the DWAI policy is always less than that under the AI policy, assuming that $I_{\mathrm{DW}}=I_{\mathrm{AI}}$. Thus, a trade-off exists between the convergence speed to fairness and the responsiveness to BW availability. The responsiveness to BW availability could be improved without affecting (increasing) the fractional packet loss $f^{\mathrm{c}}\left(S^{+}\right)$by using a larger value for $I_{\mathrm{DW}}$ than the normal one when large BW availability is detected. For instance, successive load increases may be interpreted as an indication of a non congested network and in this case a greater value for $I_{\mathrm{DW}}$ than the normal value may be applied. When network congestion is detected the normal value is used again for $I_{\mathrm{DW}}$. An example of such a multi-state scheme is presented in [19]. By using a value for $I_{\mathrm{DW}}$ greater than the normal value during a fast increase state, this multi-state DWAI-based scheme enhances the responsiveness to BW availability while retaining fractional packet loss $f^{\mathrm{c}}\left(S^{+}\right)$similar to those induced under the normal (lower) value of $I_{\mathrm{DW}}$. Thus, by introducing a multi-state scheme, the trade-off between the convergence speed to fairness and responsiveness to BW availability may be effectively addressed without increasing the fractional packet loss $f^{\mathrm{c}}\left(S^{+}\right)$.

\subsection{Smoothness and Packet Loss Rates}

In this section the better behavior of the DWAI/LDMD scheme compared to the basic AI/MD scheme in terms of smoothness and packet loss rates is discussed. The DWAI policy induces a typically diverse per flow rate increase, unlike the basic AI policy that induces the same rate increase for all flows independently from their current rate. As a consequence, the resulting rates will come closer in value after an application of the DWAI policy than of the basic AI policy, leading to a faster convergence to fairness. In addition to the previous, it is expected that the packet losses induced under the DWAI policy would be lower than those under the basic AI policy, assuming that $I_{\mathrm{DW}}=I_{\mathrm{AI}}$, since more conservative increments are applied under the former policy when the per flow rate is away from its minimum which is likely to be the case when the efficiency line is exceeded by the total load and losses occur.

Finally, the DWAI policy results in an overall smoother rate increase compared to that under the ba- sic AI policy. This is clearly the case since the increase step under the DWAI policy is typically lower than that under the basic AI policy. In fact it seems that the DWAI policy strikes a meaningful balance between smoothness and load. When the rate is low, large increase steps are allowed to bring the rate quickly to a higher level and improve quality; that is, smoothness is of secondary importance and is sacrificed to achieve quickly an overall better quality through a higher rate. On the other hand, when the rate is relatively high the DWAI policy applies a smaller increment preserving smoothness whose disturbance could harm a good quality media. The smoothness is likely to be disturbed not only due to large rate increments but mainly due to a potentially larger rate decrement that is expected to follow a large increment that leads to a higher overshoot, higher losses and finally larger decrement; the latter two impact greatly on perceptual quality.

The LDMD policy ensures that after a decrease step the total load will be adapted to a level below the efficiency line after a single action, regardless of the number of flows in the network and the level of the packet loss rate (see Proposition 5). Contrary to the LDMD policy, the basic MD policy cannot ensure such adaptation because of its fixed multiplicative decrease factor $b_{\mathrm{D}}$. If $b_{\mathrm{D}}$ is selected to be relatively large, then the rate decrease under large losses would not be sufficient and thus, more losses would occur in the next time interval(s). On the other hand, if $b_{\mathrm{D}}$ is selected to be relatively small, then the rate decrease under small losses would be unnecessarily large with the obvious impact on smoothness and throughput.

\section{Simulation Results}

\subsection{Evaluation of the DWAI/LDMD scheme}

In this section a set of simulation results are presented under the Assumptions 1 and 2 illustrating the rate adaptation behavior of the DWAI/LDMD and basic AI/MD schemes under different configuration parameters. The objective is to evaluate the DWAI/LDMD scheme with respect to smoothness, convergence to fairness and packet losses, as well as point to some intrinsic characteristics of the DWAI/LDMD scheme, such as the dynamic nature of the increase/decrease process. The considered single link network model is implemented in Matlab. In 


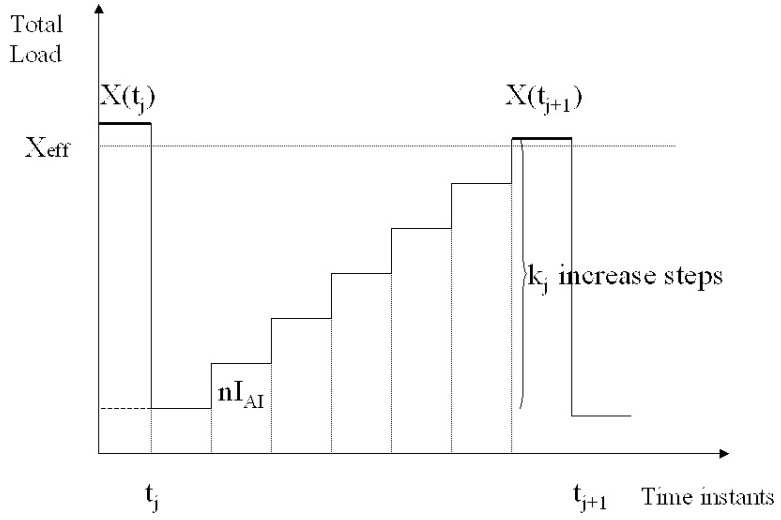

Figure 5. Rate adaptation behavior of the basic $\mathrm{AI} / \mathrm{MD}$ scheme.

all simulations the network capacity is set to $8 \mathrm{Mb} / \mathrm{s}$ and the number of flows $n$ to 12,13 and 14 for the time periods $[0,700),[700,900)$ and $[900,1000]$, respectively. The initial rate vector for the first 12 flows is given by $\vec{x}=\{m+1 *(M-m) / n, m+$ $2(M-m) / n, \cdots, M\}$ where $m=56 \mathrm{~kb} / \mathrm{s}, M=1.2$ $\mathrm{Mb} / \mathrm{s}, n=12$ and, thus, the initial total load is $X(0)=8,108,000$ bps. Flows 13 and 14 are initiated at time instants 700 and 900, respectively, with initial loads $x_{13}(700)=x_{14}(900)=600 \mathrm{~kb} / \mathrm{s}$. We selected this wide range of initial rates in order to better illustrate the convergence to fairness. Thus, in the figures that follow the behavior of flows 1 and 12 (which have the furthest apart initial rates of $151.3 \mathrm{~kb} / \mathrm{s}$ and 1.2 $\mathrm{Mb} / \mathrm{s}$, respectively) are shown.

The mean size of a single frame of a video encoded as MPEG-4 single layer at mean bitrate of 660 $\mathrm{kb} / \mathrm{s}$ (fair share for 12 flows) is $22 \mathrm{~kb}(660 \mathrm{~kb} / \mathrm{s}=30$ frames/second x $22 \mathrm{~kb} /$ frame) [30]. In all the simulations, except one, both $I_{\mathrm{DW}}$ and $I_{\mathrm{AI}}$ are set to $22 \mathrm{~kb} / \mathrm{s}$; thus the rate increment is of the order of the mean rate of a video frame. The aim is to show the superiority of the DWAI policy against the AI policy when $I_{\mathrm{DW}}$ and $I_{\mathrm{AI}}$ are equal.

In the LDMD policy, the parameter $d$ is set equal to 0.99 in order for the smoothness of the flow adaptation to be preserved, as it is required for the $\mathrm{CM}$ flows. Correspondingly, $b_{\mathrm{D}}$ is selected equal to 0.99 , 0.9845 and 0.95 for the three simulations under the

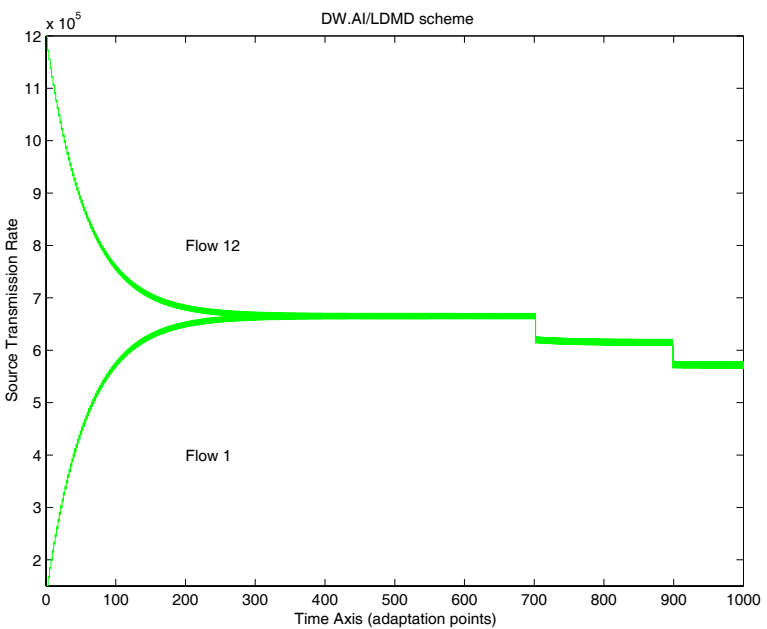

Figure 6. Rate adaptation behavior of the DWAI/LDMD scheme with $d=0.99$ and $I_{\mathrm{DW}}=22$ $\mathrm{kb} / \mathrm{s}$.

$\mathrm{AI} / \mathrm{MD}$ scheme, respectively, for a more meaningful to the comparison with the result of the DWAI/LDMD scheme. Selecting values for $b_{\mathrm{D}}$ which are less than 0.95 would lead to a less smooth adaptation behavior.

Fig. 6 shows the results under the DWAI/LDMD scheme for parameters $I_{\mathrm{DW}}=22 \mathrm{~kb} / \mathrm{s}, d=0.99$, $m=56 \mathrm{~kb} / \mathrm{s}, M=1.2 \mathrm{Mb} / \mathrm{s}$. Fig. 6 illustrates the convergence of two flows with initially very different rates to a (common) fair rate, the oscillatory behavior both during the transition period $([0, \sim 200])$ as well as during the "steady state" period ([ 200, 700]), the response of flows 1 and 12 to the introduction of flow $13([700,900])$ and to flow $14([900,1000])$. It is clear that fairness is achieved and the oscillations are fairly low. The latter may be attributed to the dynamic and "self-adjusting" rate increment that assumes a small value when flows are away from their minimum rate $m$ and to the dynamic and "self-adjusting" decrease factor $B_{\mathrm{D}}(t)=d(1-f(t))$, which assumes a large value (about $d=0.99$ ) when losses are low (typical case) leading to a small decrement.

Fig. 7 shows the results under the basic AI/MD scheme with the "equivalent" fixed parameters. That is, $I_{\mathrm{AI}}=I_{\mathrm{DW}}=22 \mathrm{~kb} / \mathrm{s}$ and $b_{\mathrm{D}}=0.99=d$; that is, when losses are very low $b_{\mathrm{D}} \approx d(1-f(t))$ and about 


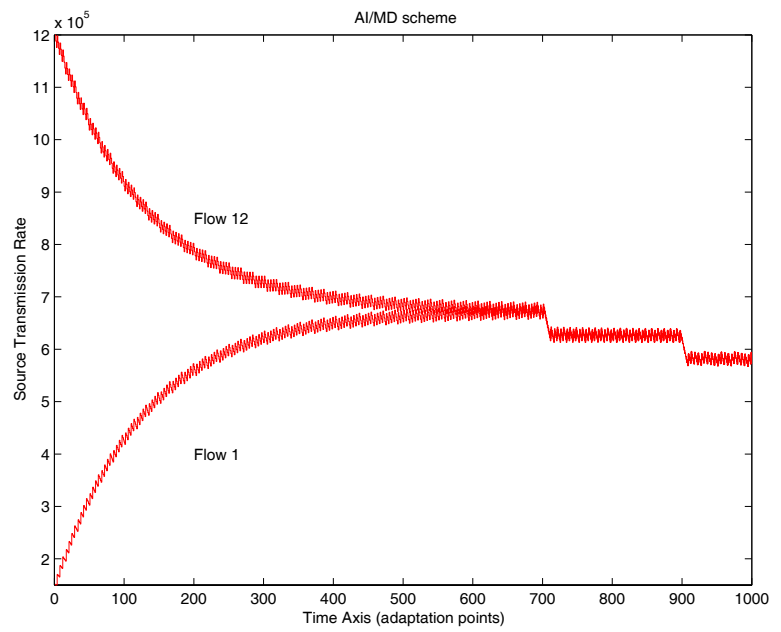

Figure 7. Rate adaptation behavior of the basic AI/MD scheme with $b_{\mathrm{D}}=0.99$ and $I_{\mathrm{AI}}=22 \mathrm{~kb} / \mathrm{s}$.

the same rate decrement would be in effect for both schemes. The idea here is to show the behavior of a scheme that is equivalent to the above DWAI/LDMD scheme where the increments and decrements are not shaped by the rate distance from $M$ and the induced losses $f(t)$, respectively.

From Fig. 6 and 7 it is observed that both the convergence speed to fairness and the oscillatory behavior are worst under the basic AI/MD scheme. The poor convergence speed to fairness is expected based on earlier discussions (see Section 3, Corollary 1), because of the application of the same increment to all flows independently from their rate as well as because of the typically larger decrease factor $b_{\mathrm{D}}=d>$ $d(1-f(t))=B_{\mathrm{D}}(t)$. The poorer oscillatory behavior under the basic $\mathrm{AI} / \mathrm{MD}$ scheme during the times that fairness has been reached may be attributed to both the inherent irregular adaptation behavior of the basic $\mathrm{AI} / \mathrm{MD}$ scheme and the larger increment under the basic AI policy, that leads to higher overshoots. These higher overshoots - along with the smaller decrement under large packet losses - is responsible for the induced higher mean conditional packet loss rate under the basic AI/MD scheme. Specifically, these loss rates are $1.53 \%(0.55 \%), 2.00 \%(0.84 \%), 2.11 \%(1.12 \%)$ under the basic AI/MD (DWAI/LDMD) scheme, over the periods $[0,700],[700,900]$ and $[900,1000]$, respectively. The mean conditional packet loss rate is defined as the long-term average of the following quantity defined only over periods with non zero packet losses: (number of packets lost over the current loss report period)/(number of packets sent over the current loss report period). The above simulation results point to the advantages of a scheme that adapts dynamically its key parameters in a meaningful way (DWAI/LDMD) over a scheme that does not (AI/MD). The remaining results emphasize some key trade-offs in the selected values of key parameters and the fact that a static parameter cannot effectively balance competing objectives.

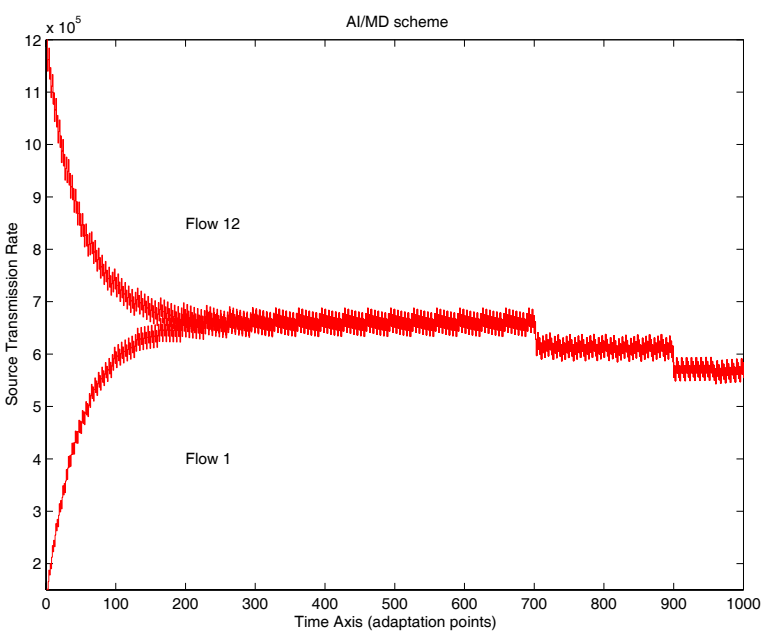

Figure 8. Rate adaptation behavior of the basic AI/MD scheme with $b_{\mathrm{D}}=0.95$ and $I_{\mathrm{AI}}=22 \mathrm{~kb} / \mathrm{s}$.

Fig. 8 shows the results under the $\mathrm{AI} / \mathrm{MD}$ scheme with a lower decrease factor $b_{\mathrm{D}}=0.95$ (and $I_{\mathrm{AI}}=22$ $\mathrm{kb} / \mathrm{s})$. This decrease factor would lead to a larger diversification of the decrements that would be applied to flows of different rates (larger decrement to higher rates) and thus improve the convergence speed to fairness (compared to $b_{\mathrm{D}}=0.99$ ). This indeed is observed to be the case. In fact $b_{\mathrm{D}}=0.95$ brings the convergence speed to fairness under the basic AI/MD scheme to almost match that under the DWAI/LDMD 


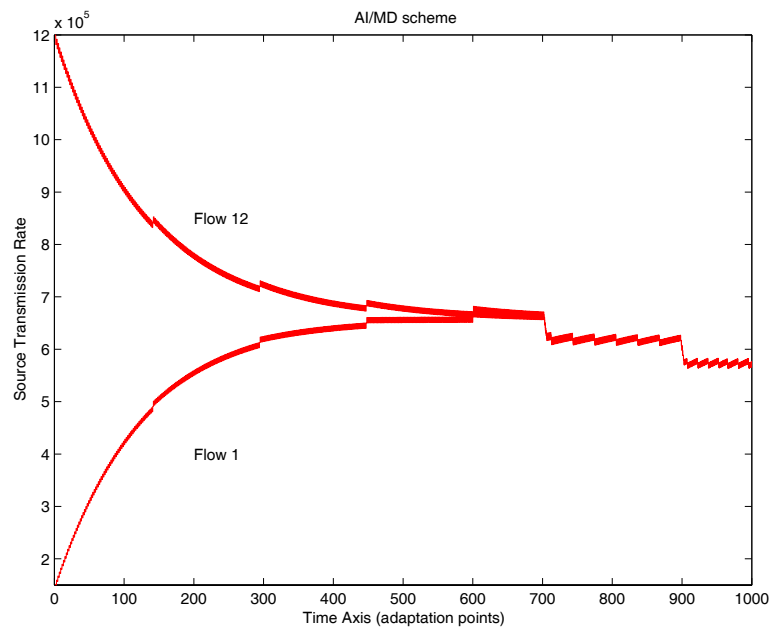

Figure 9. Rate adaptation behavior of the basic $\mathrm{AI} / \mathrm{MD}$ scheme with $b_{\mathrm{D}}=0.9845$ and $I_{\mathrm{AI}}=$ $\frac{M-\frac{X_{\text {eff }}}{n}}{M-m} I_{\mathrm{DW}}$.

scheme for the parameters considered in Fig. 6. The price paid for this improvement (compared to the case for $b_{\mathrm{D}}=0.99$ ) are larger oscillations (less smooth rates). In Fig. 8 it is also illustrated the irregular adaptation behavior of the flows under the basic AI/MD scheme for the periods $[0,700],[700,900]$. The induced conditional packet loss rates are higher than those under the DWAI/LDMD scheme. Specifically the former are equal to $2.1 \%, 2.04 \%$ and $4.04 \%$ over the periods $[0,700],[700,900]$ and $[900,1000]$, respectively.

In Fig. 9 a lower fixed increment is selected that is about equal to the increment applied by the DWAI/LDMD scheme in Fig. 6 when the flows have reached the fairness line (period $[\sim 200,700])$. That is, $I_{\mathrm{AI}}=\frac{M-\frac{X_{\text {eff }}}{n}}{M-m} I_{\mathrm{DW}}$. Also, the parameter $b_{\mathrm{D}}$ is selected to be equal to 0.9845 which is the value of $B_{\mathrm{D}}(t)$ that is applied by the DWAI/LDMD scheme in Fig. 6 during the period $[0,700]$. Therefore, the flows under the basic AI/MD scheme present similarly smooth adaptation behavior as the flows under the DWAI/LDMD scheme, as the former flows converge to the fairness line; that is the flows present similar rate increments and decrements (see Fig. 10,

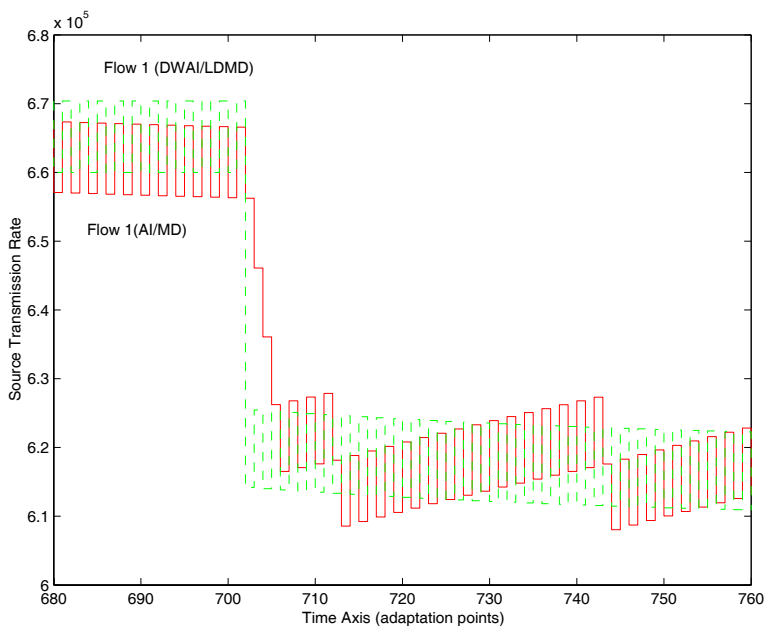

Figure 10. Similar adaptation behavior of the DWAI/LDMD (dashed lines) and basic AI/MD schemes with $d=0.99, b_{\mathrm{D}}=0.9845$ and $I_{\mathrm{AI}}=$ $\frac{M-\frac{X_{\text {eff }}}{n}}{M-m} I_{\mathrm{DW}}$.

period $[685,700])$. The idea here is to illustrate that even if the parameters of the basic AI/MD scheme are properly selected to provide a smooth behavior, the smoothness is retained only for the specific network conditions.

Indeed after the initiation of a new flow at time instant 700, the flows under the DWAI/LDMD continue to retain their smooth adaptation behavior because of DWAI/LDMD scheme's "self-adjusting" property, whereas the flows under the basic AI/MD scheme do not retain their smoothness but present irregular behavior in the sequel. Fig. 10 also illustrates that the DWAI/LDMD scheme adapts in a single decrease step, protecting the flow from packet losses in the next periods (as discussed earlier) whereas the basic $\mathrm{AI} / \mathrm{MD}$ scheme requires a sequence of decrease steps.

\subsection{Evaluation of the DWAI/LDMD scheme in re- alistic environments}

The simulation results presented above have been obtained assuming that Assumptions 1 and 2 hold. As this may not be practically the case, further simulations have been carried out in a more realistic environment based on the ns- 2 simulator. The network 
models of single-hop and chain topology, depicted in Fig. 11 and 12 , respectively, are implemented in the ns-2 simulator.

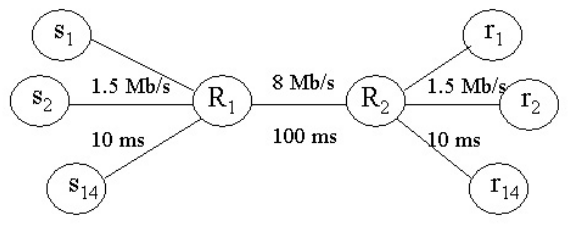

Figure 11. Single-hop topology

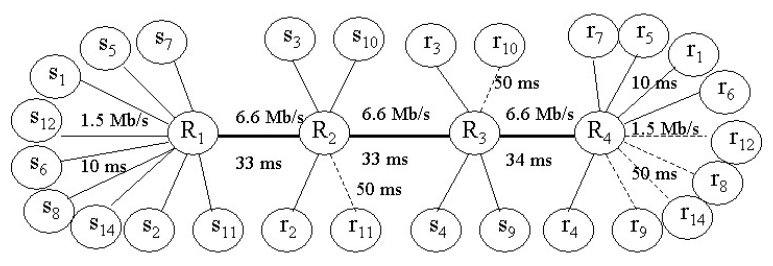

Figure 12. Chain network topology.

The considered environment consists only of adaptable CM (RTP/UDP/IP) flows, that is, it is free of TCP flows, as it may be the case in a differentiated services environment. Specifically, RTP/UDP/IP flows with packets of size of 1000 bytes and drop tail routers with buffers of size of 100 packets have been considered. The drop tail routers with small buffers (FIFO queuing) tend to distribute losses in proportion to the flows' rates when the packets are small compared to the capacity of the link. Hence, Assumption 2 is fulfilled. RTCP provides the source with the packet loss rate feedback through the RTCP receiver reports (RR) reporting the numbers of lost and sent packets over the last time interval (packet loss rate $f$ is computed as \# of lost packets / \# of sent packets). Flows 1-12 are either initiated at time instant 0 or during the first five seconds but at different and randomly selected time instants, according to one of the considered simulation scenarios defined in the sequel. Flows 13 and 14 in all simulations are initiated at the $500^{\text {th }}$ and $700^{t h}$ adaptation points which occur at second 2500 and 3500, respectively. The overall simulated period has a duration of 4,000 seconds. The following four scenarios are implemented.

(a) Scenario A: Flows 1-12 are initiated at time instant 0 . RTCP RRs are sent regularly every 5 seconds. The round trip time is common for all flows, therefore the feedback is synchronized. The single-hop topology is considered.

(b) Scenario B: Flows 1-12 are initiated during the first five seconds but at different times randomly determined. RTCP RR are sent not at regular intervals (every 5 seconds) but every $5 \pm 1.5$ seconds, randomly determined, therefore the feedback is non-synchronized. The single-hop topology is considered.

(c) Scenario C: As scenario B, but for different round trip times. The link delays between the router R2 and receivers $\{r 1-r 6, r 13\}$ and $\{r 7-r 12, r 14\}$ are set to 10 and $50 \mathrm{~ms}$, respectively; therefore the RTT for flows $1-6,13$ and $7-12,14$ are $240 \mathrm{~ms}$ and $320 \mathrm{~ms}$, respectively.

(d) Scenario D: As scenario C, but for the chain network topology depicted in Fig. 12. The round trip times of the flows are different and the feedback non-synchronized.

In the simulations of Fig. 13 the DWAI/LDMD scheme has been employed with $I_{\mathrm{DW}}=22 \mathrm{~kb} / \mathrm{s}$ and $d=0.99$, whereas in the simulations of Fig. 14 the basic AI/MD scheme has been employed with $I_{\mathrm{AI}}=22 \mathrm{~kb} / \mathrm{s}$ and $b_{\mathrm{D}}=0.9845$ which is equal to $B_{\mathrm{D}}(t)$ applied by the DWAI/LDMD scheme (see Fig. 6 and associated simulation results). In all figures, only the adaptation behavior of flows 1 and 12 under the considered scheme are shown. 


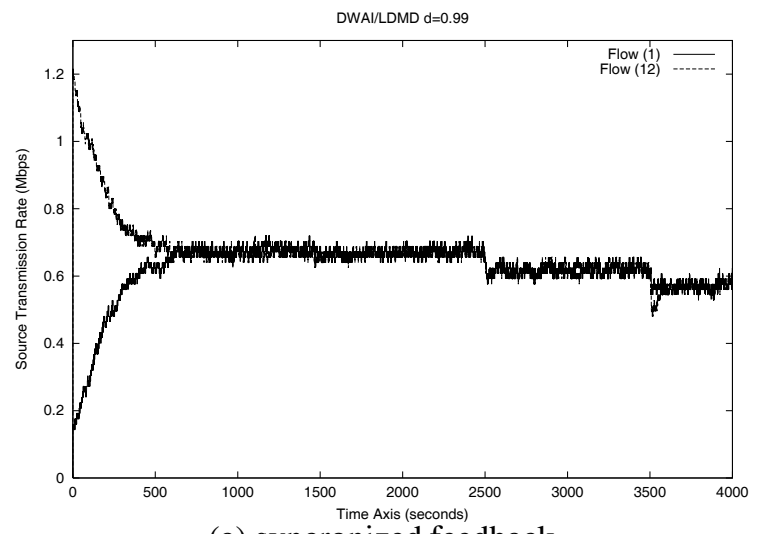

(a) syncronized feedback

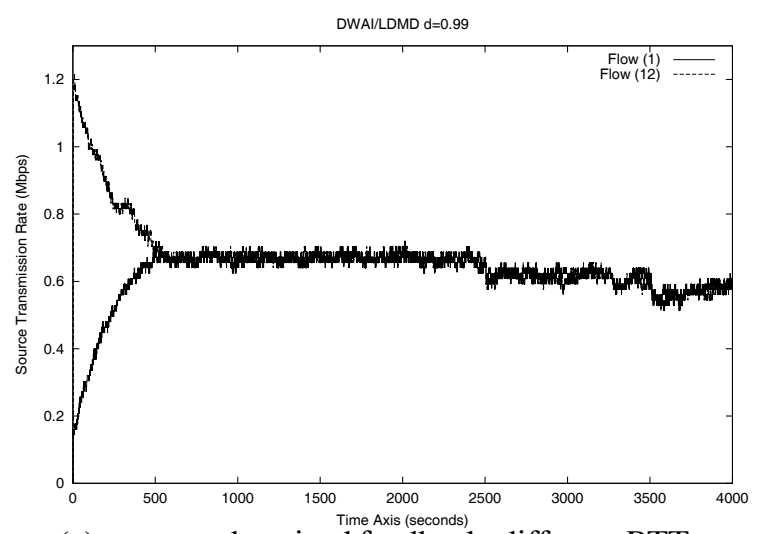

(c) non-synchronized feedback, different RTTs

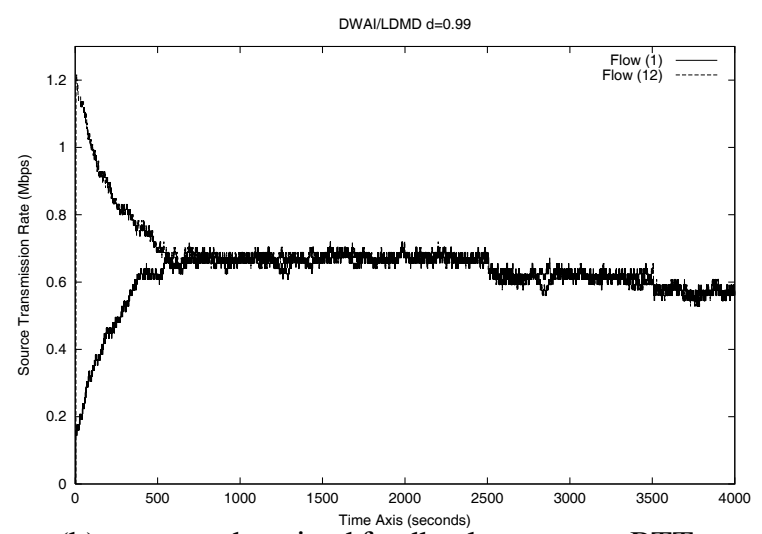

(b) non-synchronized feedback, common RTTs

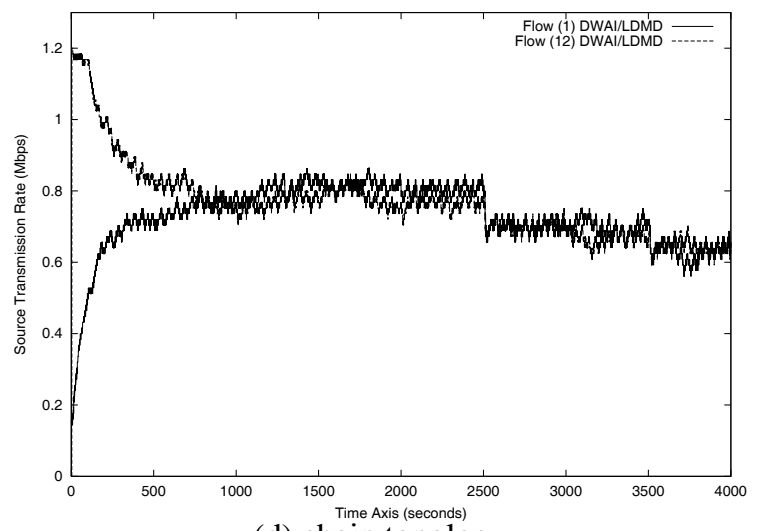

(d) chain topology

Figure 13. Rate adaptation behavior of the DWAI/LDMD scheme with $d=0.99$ and $I_{\mathrm{DW}}=22 \mathrm{~kb} / \mathrm{s}$.

The coefficient of variance ( $\mathrm{CoV})$ is used as a metric for quantifying the shorter-term smoothness. $\mathrm{CoV}$ is computed for each flow with one sample per second over the time interval $[1000,2500]$. Column $\overline{\mathrm{CoV}}$ in Table 1 illustrates the average of $\mathrm{CoV}$ for all flows. The mean size of oscillation $(\bar{O})$ around the fair share, when the fair share has been reached, is defined as a second metric for quantifying the longerterm smoothness. $\bar{O}$ is defined as follows:

$\bar{O}=\frac{\sum_{i=1}^{n} \sum_{t=1000}^{4000}\left|x_{i}(t)-x_{\text {fair }}(t)\right|}{n(4000-1000)}$.

Clearly, the longer-term smoothness is improved as $\bar{O} \rightarrow 0$.

Fig. 13 and 14 show the results under the DWAI/LDMD and basic AI/MD schemes for the four aforementioned scenarios. These figures illustrate that the adaptation behavior is smoother under the DWAI/LDMD scheme than that under the basic $\mathrm{AI} / \mathrm{MD}$ scheme. Indeed, in addition to the visual comparison of figures, the values of the metrics of $\overline{C o V}$ and $\bar{O}$ shown in Table 1 are lower under the DWAI/LDMD schemes.

Also, Fig. 15 (a) and (b) show that the flows converge faster to the fair share under the DWAI/LDMD scheme. This holds for all the simulation scenarios. It should be noted that both the DWAI/LDMD and basic AI/MD scheme converge to the fair share under different RTTs, while it is known that TCP is unfair under heterogeneous feedback.

The TCP flows (small time scale feedback) are more sensitive than the RTCP-based DWAI/LDMD 


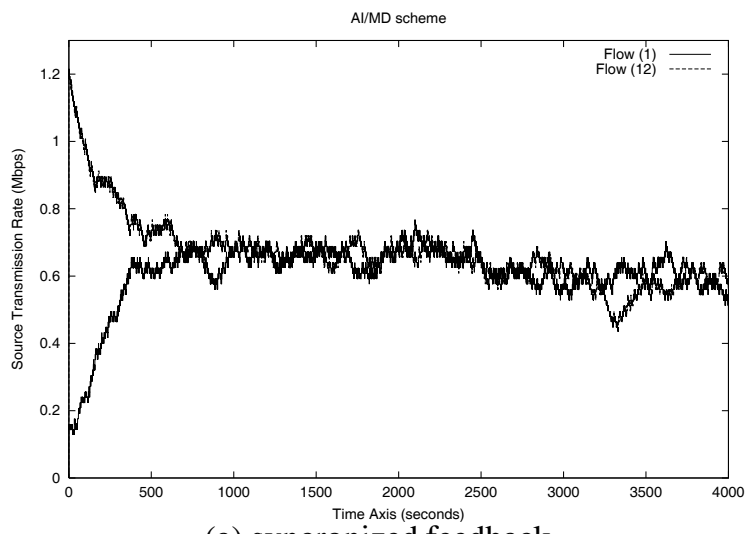

(a) syncronized feedback

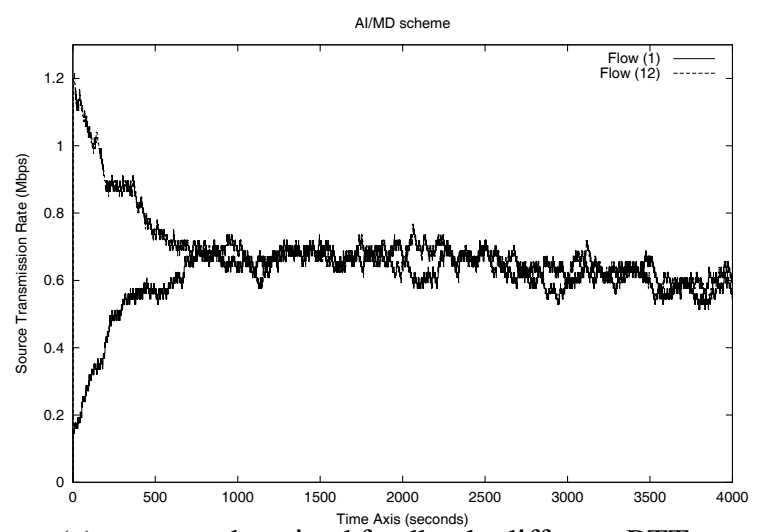

(c) non-synchronized feedback, different RTTs

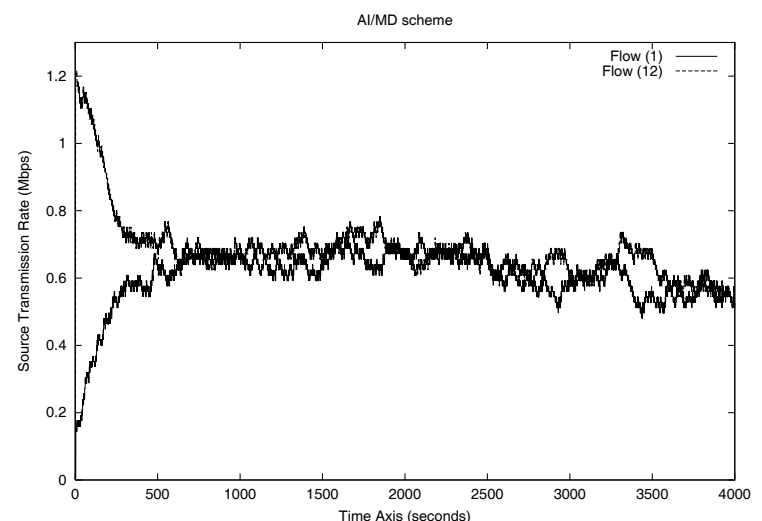

(b) non-synchronized feedback, common RTTs

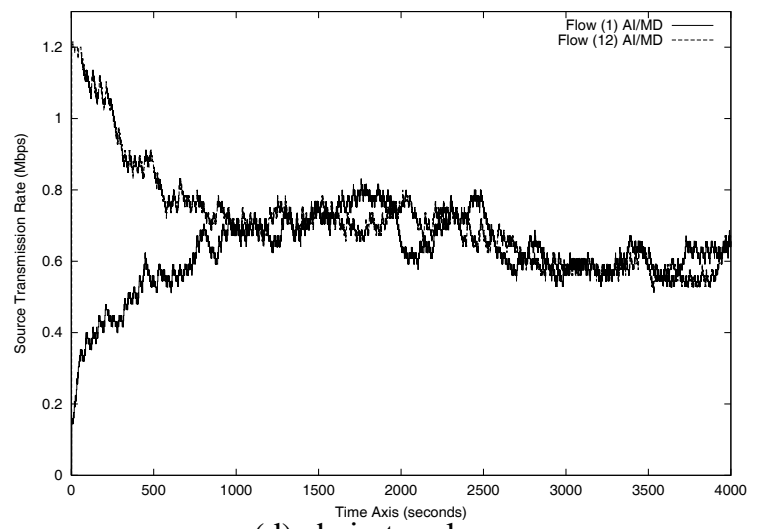

(d) chain topology

Figure 14. Rate adaptation behavior of the basic AI/MD scheme with $b_{\mathrm{D}}=0.9845$ and $I_{\mathrm{AI}}=22 \mathrm{~kb} / \mathrm{s}$.

and $\mathrm{AI} / \mathrm{MD}$ flows (longer time scale feedback) in heterogeneously delayed feedback. TCP is a windowsbased AI/MD protocol that receives feedback on RTT time scale. So, TCP flows that experience very different RTTs receive very different delayed feedback that captures network status at different times. This leads to diverse network status view and diverse increase or decrease actions that eventually result in unfair shares. On the other hand, the DWAI/LDMD and basic AI/MD schemes considered in this paper are ratebased with RTCP-based feedback. Since the feedback includes information about a larger time interval than RTT, the sources obtain a more common (averaged) view about the current network status than the TCP sources and react more consistently and, thus, like synchronized, achieving fair shares.
The number of lost packets, the long-term packet loss rate (total number of packets lost / total number of packets sent) and the mean conditional packet loss rates induced over the entire simulation period $([0,4000])$ are lower under the DWAI/LDMD scheme (see columns lost packets, $l t \overline{P L R}$ and $m c \overline{P L R}$, respectively, in Table 1).

The sequence of the reported packet loss rates immediately after the initiation of flow 13 at time 2500 are $0.91 \%, 7.76 \%, 7.78 \%, 5.36 \%, 3.28 \%, 0.45 \%$, $1.87 \%, 0 \%$ for flow $1,0.33 \%, 6.75 \%, 5.82 \%, 3.00 \%$, $4.17 \%, 0.78 \%, 0.28 \%, 0 \%$ for flow 12 under the basic AI/MD scheme, and $4.57 \%, 6.68 \%, 0 \%$ for flow $1,5.76 \%, 2.18 \%, 0 \%$ for flow 12 under the DWAI/LDMD scheme in scenario B. In all scenarios, the DWAI/LDMD scheme adapts in fewer decrease 


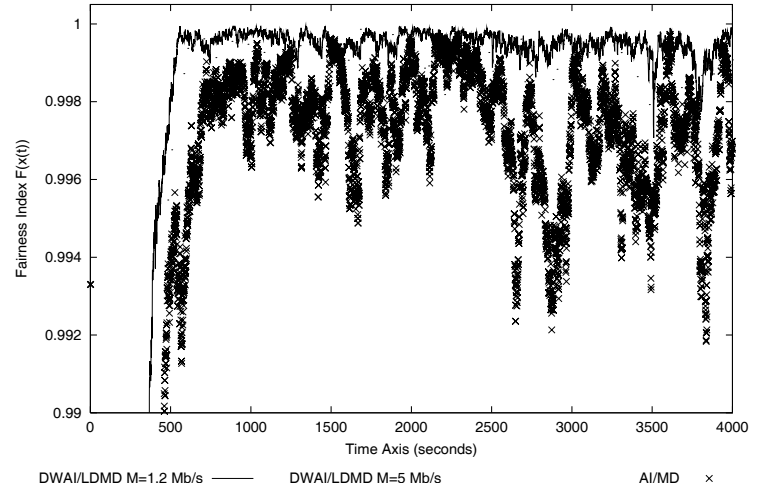

DWA/LDMD M=1.2 Mb/s
(a) non-synchronized feedback, common RTTs

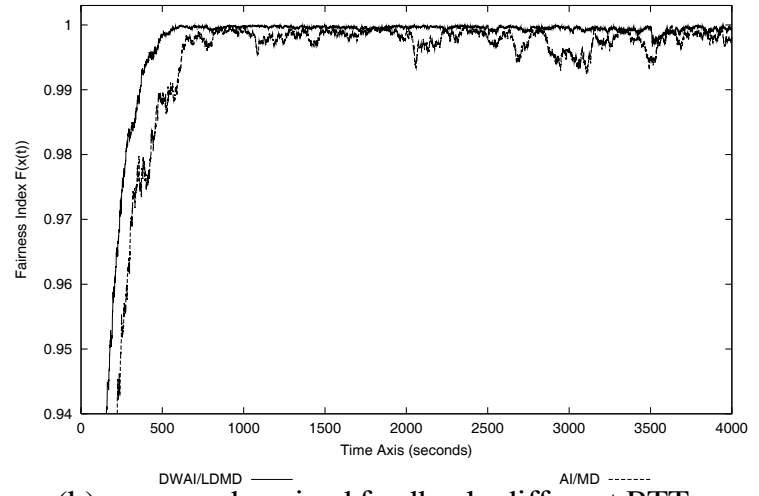

(b) non-synchronized feedback, different RTTs

Figure 15. Fairness indices under scenarios B and C scenarios.

steps, whereas the basic AI/MD scheme requires a sequence of decrease steps during which several packet losses occur. Finally, the mean throughput is higher under the DWAI/LDMD scheme than under the basic AI/MD scheme in all scenarios (see the corresponding column $\overline{T h r}$ in Table 1).

Fig. 16 and Table 1 (row 5) show the results under the DWAI/LDMD scheme with $M=5 \mathrm{Mb} / \mathrm{s}$ (as opposed to $M=1.2 \mathrm{Mb} / \mathrm{s}$ in earlier figures), a relatively large value compared to the fair share which is around $0.6 \mathrm{Mb} / \mathrm{s}$. This scheme presents better smoothness and fairness than the basic AI/MD scheme (see Table 1 and Fig. 15). Therefore, even for large $M$, the DWAI/LDMD scheme continues to exhibit different (and better) behavior than the basic AI/MD scheme and is not reduced to the basic AI/MD scheme.

In the sequel, simulation results under the TCPcompatible schemes TCP, TFRCP and binomial $\operatorname{IIAD}(1,2 / 3)$ - see Section 7 on related work - are shown and compared against the results under the DWAI/LDMD scheme. The single-hop network model of Fig. 11 is considered. Flows 1-12 are randomly initiated during the first 0.5 seconds. Flows 13 and 14 are initiated at time instants 2500 and 3500 seconds, respectively. Fig. 17, 18 and 19 show the adaptation behavior of flow 1 under the TCP, TFRC and $\operatorname{IIAD}(1,2 / 3)$ protocols, respectively, in an environment where only TCP, TFRC or IIAD flows exist. Fig. 17 clearly illustrates that TCP is not suit-

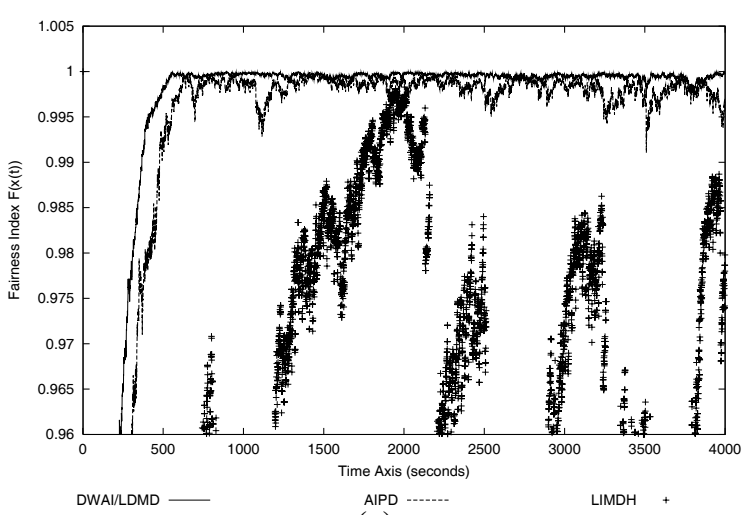

(a)

Figure 21. Fairness indices of DWAI/LDMD, AI/PD and LIMDH schemes.

able for CM streaming due its large rate variation. The TFRC and IIAD flows are smoother (Fig. 18 and 19) than the TCP flow (Fig. 17), but the rate variation is still high for $\mathrm{CM}$ streaming (see also column $\overline{C o V}$ in Table 2). Therefore, it is concluded that for the considered environment, where $\mathrm{CM}$ flows are isolated from data flows, the DWAI/LDMD scheme with $d=0.99$ is more suitable than the TFRC and $\operatorname{IIAD}(1,2 / 3)$ schemes (compare Fig. 13 with 18 and 


\begin{tabular}{|c|l|c|c|c|c|c|c|c|}
\hline & Schemes/Metrics & $d$ or $b_{\mathrm{D}}$ & $l t \overline{P L R}$ & $m c \overline{P L R}$ & \# lost packets & $\overline{C o V}$ & $\bar{O}(\mathrm{~kb} / \mathrm{s})$ & $\overline{T h r}$ \\
\hline \hline 1. & DWAI/LDMD sync. (a) & 0.99 & $0.498 \%$ & $1.04 \%$ & 20,026 & 0.0249 & 12.21 & 0.9950 \\
\hline 2. & AI/MD sync. (a) & 0.9845 & $0.857 \%$ & $1.11 \%$ & 34,591 & 0.0408 & 28.68 & 0.9914 \\
\hline \hline 3. & DWAI/LDMD non-sync. (b) & 0.99 & $0.423 \%$ & $0.819 \%$ & 17,000 & 0.0242 & 12.12 & 0.9957 \\
\hline 4. & AI/MD non-sync. (b) & 0.9845 & $0.662 \%$ & $0.856 \%$ & 26,661 & 0.0429 & 25.41 & 0.9933 \\
\hline 5. & $\begin{array}{l}\text { DWAI/LDMD non-sync. (b) } \\
\text { M }\end{array}$ & 0.99 & $0.649 \%$ & $1.06 \%$ & 26,139 & 0.0351 & 18.07 & 0.9935 \\
\hline \hline 6. & DWAI/LDMD diff. RTTs (c) & 0.99 & $0.427 \%$ & $0.81 \%$ & 17,171 & 0.0235 & 12.24 & 0.9957 \\
\hline 7. & AI/MD diff. RTTs (c) & 0.9845 & $0.688 \%$ & $0.892 \%$ & 27,712 & 0.0423 & 24.05 & 0.9931 \\
\hline
\end{tabular}

Table 1

Comparison of AI/MD and DWAI/LDMD schemes.

\begin{tabular}{|l|l|c|c|c|c|c|c|}
\hline & Schemes/Metrics & $l t \overline{P L R}$ & sent packets & \# lost/retransmitted packets & $\overline{C o V}$ & $\overline{T h r}$ & $\overline{N U}$ \\
\hline \hline 1. & TCP & $10.7 \%$ & $3,511,702$ & 376,706 & 0.5599 & 0.892 & 0.972 \\
\hline 2. & TFRC & $0.221 \%$ & $4,004,822$ & 8,881 & 0.1782 & 0.997 & 1 \\
\hline 3. & IIAD $(1,2 / 3)$ & $2.64 \%$ & $3,844,616$ & 101,514 & 0.2166 & 0.973 & 0.986 \\
\hline
\end{tabular}

Table 2

Comparison of TCP-compatible schemes.

\begin{tabular}{|l|l|c|c|c|c|c|c|c|}
\hline & Schemes/Metrics & $l t \overline{P L R}$ & $m c \overline{P L R}$ & \# lost packets & $\overline{C o V}$ & $\bar{O}(\mathrm{~kb} / \mathrm{s})$ & $\overline{T h r}$ & $\overline{N U}$ \\
\hline \hline 1. & DWAI/LDMD $d=0.99$ & $0.423 \%$ & $0.819 \%$ & 17,000 & 0.0220 & 12.12 & 0.9957 & 1 \\
\hline 2. & AI/PD $\beta=2$ & $0.792 \%$ & $1.35 \%$ & 31,927 & 0.0423 & 22.82 & 0.9920 & 1 \\
\hline 3. & LIMDH & $0.415 \%$ & $1.29 \%$ & 16,397 & 0.1223 & 83.44 & 0.9958 & 0.987 \\
\hline 4. & AI/MD $(k=0, l=1)$ & $0.662 \%$ & $0.856 \%$ & 26,661 & 0.0408 & 25.41 & 0.9933 & 1 \\
\hline
\end{tabular}

Table 3

Comparison of DWAI/LDMD with other rate-based schemes.
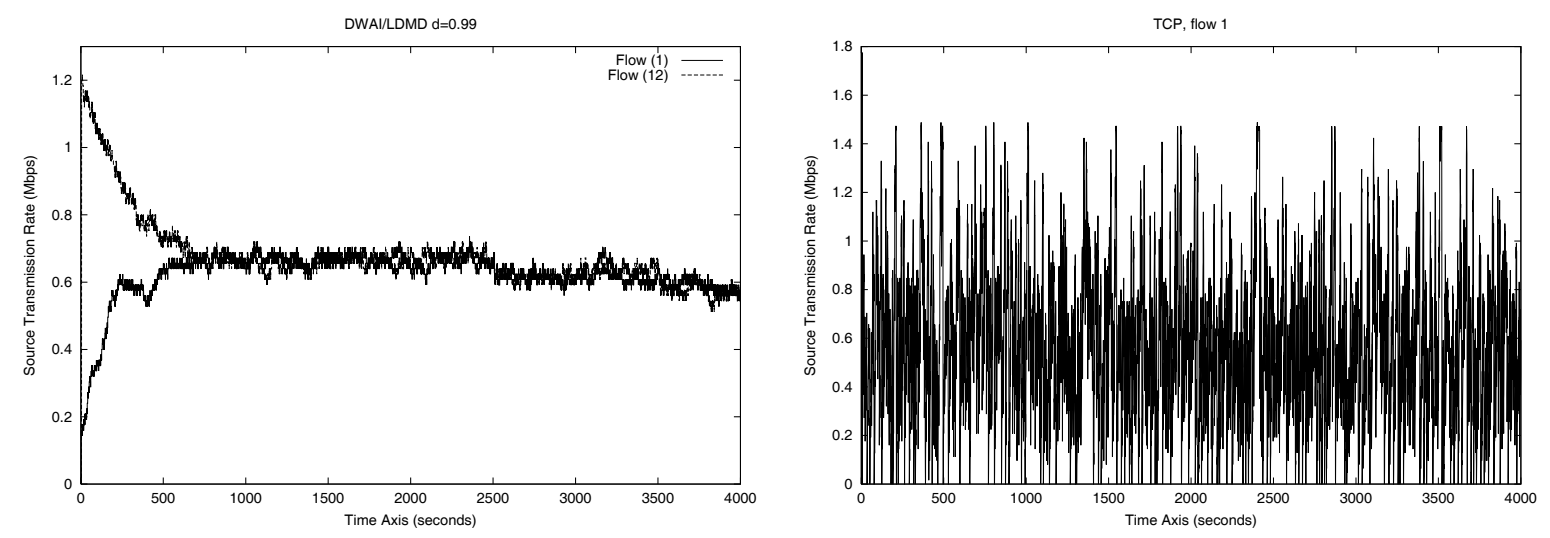

Figure 16. DWAI/LDMD scheme with $M=5 \mathrm{Mb}$. . Figure 17. Rate adaptation behavior of TCP. 


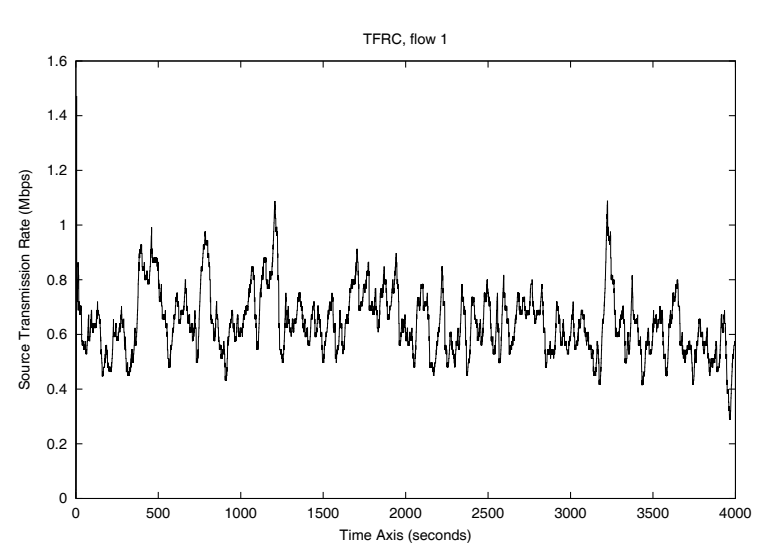

Figure 18. Rate adaptation behavior of TFRC.

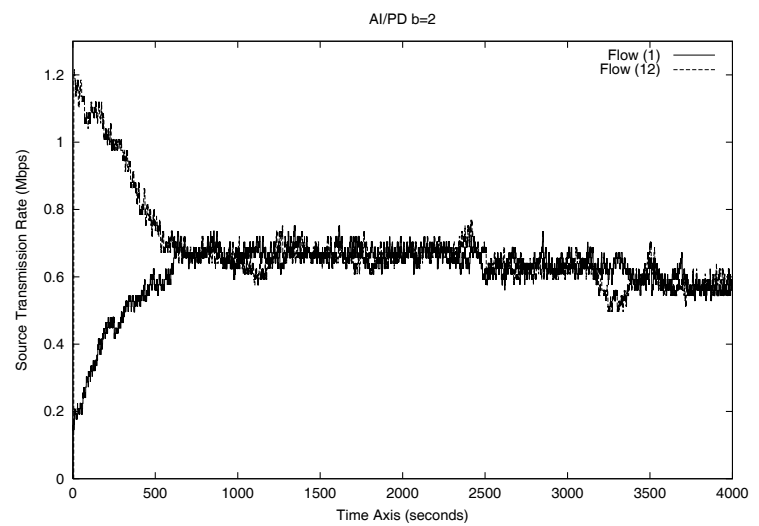

(a)

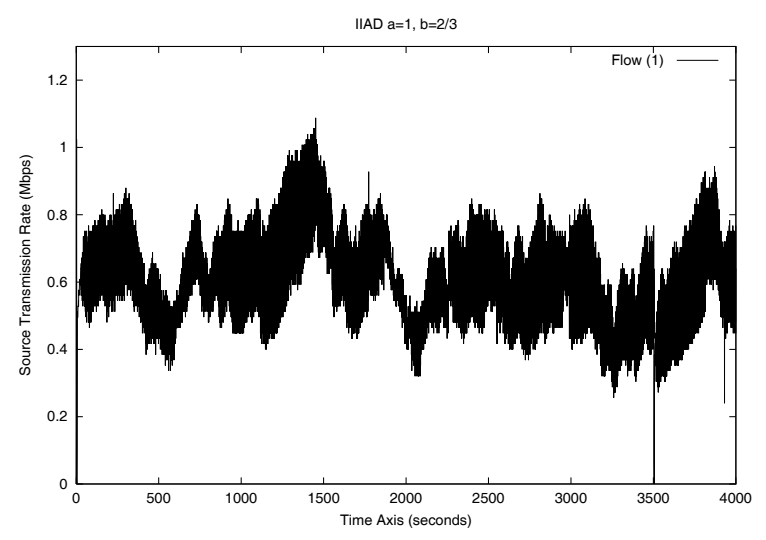

Figure 19. Rate adaptation behavior of $\operatorname{IIAD}(1,2 / 3)$ scheme.

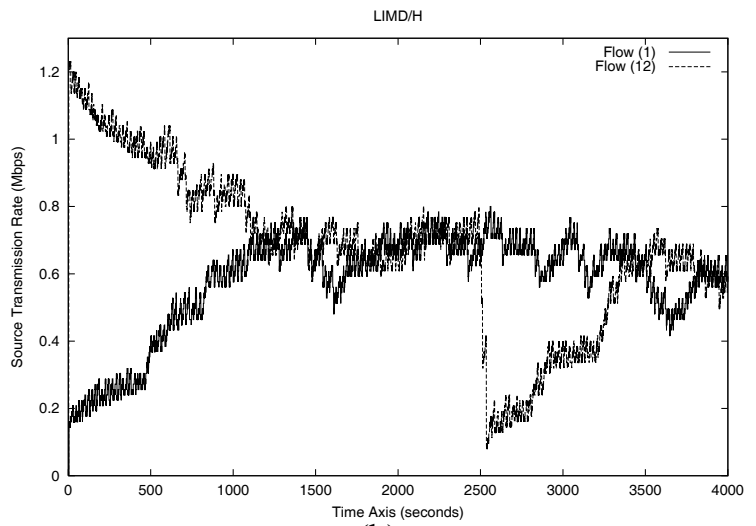

(b)

Figure 20. Rate adaptation behavior of the AI/PD and LIMDH schemes with $I_{\mathrm{AI}}=22 \mathrm{~kb} / \mathrm{s}$.

Table 1 with 2).

In the sequel, simulation results under the RTCPbased non-AI/MD schemes AIPD, LIMDH - see Section 7 on related work - are shown and compared against the results under the DWAI/LDMD scheme. Scenario B is considered for the simulations. Flows 13 and 14 are initiated at time instants 2500 and 3500 seconds, respectively. These schemes are implemented as rate-based schemes with RTCP-based feedback.

Fig. 20 (a) and (b) show the results under the
AI/PD with $\beta=2$ and LIMDH schemes, respectively. Clearly, the adaptation behavior under these schemes are not as smooth as that under the DWAI/LDMD scheme (compare Fig. 20 with Fig. 13 and the metrics $\overline{\mathrm{CoV}}$ and $\bar{O}$ in Table 3). It should be noted that flows under the LIMDH scheme may dramatically decrease their rate in case of successive losses due to the exponential increase of the parameter $d$ in the new rate $=$ $\bar{R}(1-d \beta)$, where $\bar{R}$ is the average sending rate and $\beta=0.05$ (see Section "Related Work"). This is illustrated in Fig. 20 (b) (flow 1) and Fig. 21 (a) by 
the fairness index that corresponds to LIMDH, which presents dramatic decreases.

The flows under these schemes converge slower to the fair share than under the DWAI/LDMD scheme (see the corresponding fairness indices in Fig. 21 (a)), especially the convergence to fair share under the LIMDH scheme is very slow. Regarding the throughput, it is concluded from column $\overline{T h r}$ in Table 3 that the throughput under the AI/PD scheme is lower than the throughput under the DWAI/LDMD scheme, whereas the throughput under the LIMDH scheme is more or less similar to that under the DWAI/LDMD scheme. It should be noted, though, that the network utilization under the LIMDH scheme is lower than other schemes. This is attributed to the fact that the LIMDH scheme does not respond fast to bandwidth fluctuations since the average and not the current transmission rate is taken into account when the new rate is shaped.

The results of the simulations carried out in ns2 have evaluated the behavior of the DWAI/LDMD scheme against those of the basic AI/MD scheme and other non AI/MD schemes in terms of smoothness, convergence to fairness, packet losses and throughput in realistic environments (different round trip times and non-synchronized feedback).

\section{Related Work}

\section{1. $A I / M D$ and TCP}

The congestion control mechanism of TCP [2] is based on the Additive Increase Multiplicative Decrease (AI/MD) algorithm [1,3], a linear control algorithm. In the linear control algorithms the flows adjust their rate to a value that is determined by a portion of the current rate plus a fixed amount as shown below, depending on whether a binary feedback $f$ indicates congestion $(f>0)$ or not $(f=0)$

new rate $= \begin{cases}a_{\mathrm{I}}+b_{\mathrm{I}}(\text { current rate }) & \text { if } f=0 \\ a_{\mathrm{D}}+b_{\mathrm{D}}(\text { current rate }) & \text { if } f>0\end{cases}$

The convergence to efficiency and fairness and the distributedness properties of linear control functions are discussed in [3], under the assumptions that all users: (a) receive the binary feedback simultaneously (synchronized feedback); (b) receive the same feedback; (c) react to the feedback in the same manner; Assumptions (a), (b) and (c) are widely adopted for the study of congestion control schemes $[20,18,28,24]$ and hold in this paper, as well. Chiu and Jain have shown analytically in [3] that the linear control functions with parameters $a_{\mathrm{I}}>0, b_{\mathrm{I}}=1, a_{\mathrm{D}}=0,0<$ $b_{\mathrm{D}}<1$, satisfy the requirements for convergence to efficiency and fairness. Such linear control functions implement a basic Additive Increase Multiplicative Decrease scheme (AI/MD). The basic AI/MD scheme may be applied to both window and rate based transmission schemes. TCP is a basic window-based $\mathrm{AI} / \mathrm{MD}$ scheme with $b_{\mathrm{D}}=0.5$ that implements positive acknowledgement per packet, therefore its control loop delay is equal to round trip time (RTT).

\subsection{TCP-compatible schemes}

In a single-class network, like the best-effort Internet, it is conceivable that flows with different requirements implement different congestion control schemes. Nevertheless, it is strongly desirable that resources are fairly allocated under the different congestion control schemes. This is the case in an environment where TCP and the equation-based congestion control schemes proposed in [23] co-exist. The latter approach attempts to provide for a smoother adaptation behavior (required for instance by $\mathrm{CM}$ applications) while achieving a throughput similar to that of a TCP flow (that is, it is TCP-compatible) by employing the TCP flows' throughput estimation formula derived in [4]. Protocols that follow the aforementioned formula to estimate a rate friendly to TCP flows are characterized as TCP-friendly. This formula is based on the loss event probability and RTT and therefore the estimation of the TCP-friendly rate is susceptible to inaccuracies in the measurement of the loss probability and RTT. TFRCP [16] is an example of window-based TCP-friendly control schemes. In the TFRC protocol the receiver calculates the packet loss rate and sends a feedback, that includes the loss rate, to the sender once every RTT. RTT is estimated by the sender. The Rate Adaptation Protocol (RAP) [15] is a rate-based protocol that uses the AI/MD control scheme for shaping the sending rate. RAP acknowledges each packet having a control loop of the RTT time scale. It appears that RAP is TCPcompatible without using Padhye's formula [4].

Bansal and Balakrishnan have introduced in [20] a new class of nonlinear congestion control schemes referred to as binomial and elaborate on in- 
crease/decrease rules that enable TCP-compatibility without the need for loss-rate tracking as in [16] and generally in the equation-based congestion control scheme approach [23]. In binomial schemes the flows adjust their load according to the following:

new rate $= \begin{cases}\text { curr. rate }+a_{\mathrm{I}} /(\text { curr. rate })^{k} & \text { if } f=0 \\ \text { curr. rate }-\beta(\text { curr. rate })^{l} & \text { if } f>0\end{cases}$

where $a_{\mathrm{I}}>0$ and $0<\beta<1$. They have shown that a window-based binomial congestion control scheme converges to fairness if $k+l>0$, and is TCPcompatible if $k+l=1$. For $k=0, l=1$ the basic linear AI/MD scheme is obtained $\left(\beta=1-b_{\mathrm{D}}\right)$. The two specific algorithms presented there (Inverse Increase Additive Decrease (IIAD, $k=1, l=0$ ) and Square Root (SQRT, $k=l=\frac{1}{2}$ )) are shown to be TCP-compatible and smoother than TCP for specific increase and decrease parameters. However, in the presence of high loss rate, they do not retain their smoothness unlike under the scheme proposed in this paper.

The AI/MD and binomial schemes are memoryless, that is, they do not utilize any history information. In [28] a spectrum of TCP-friendly windowbased schemes that use history information in the increase policy is presented. The increase policy of the binomial class is modified to take into consideration the window size after the last decrease (history information), and the values of $k$ and $l$ are restricted to be $k>-1$ and $l \leq 1$. The Square Increase Multiplicative Decrease scheme [27] (SIMD, $k=-\frac{1}{2}$, $l=1$ ) is used as a study case. In SIMD, the congestion window increases super-linearly, in proportion to the square of the time elapsed since the detection of the last loss event, resulting in highly aggressive behavior and fast convergence to fairness.

The proposed DWAI/LDMD scheme is not TCPcompatible and its feedback mechanism is RTCPbased since we assume that in the future Internet the CM flows will be based on RTP/UDP and be isolated from the TCP flows. However, future work includes the investigation of a TCP-compatible variation of the proposed scheme. Some of the first experiences on the dynamic control of flows based on RTP/RTCP are presented in [8,9]. The schemes presented in [10][14] are examples of rate-based TCP-friendly control schemes that use an RTCP-based feedback mechanism. Recall that in the RTCP-based feedback mecha- nisms the interval between two feedbacks is of larger time scale than RTT, the nature of the adaptation is non self-clocking and the feedback is of continuous value (non-binary). We show in the present paper that the flow adaptation behavior under schemes with RTCP-based feedback could be smoother than the TCP-compatible schemes TFRC and IIAD.

\subsection{Loss Dependent Decrease based Schemes}

The congestion control schemes utilize feedback to decide on the proper increase or decrease action. In the case of continuous feedback, the loss rate can be used by the decrease policy to determine the new (decreased) rate. ${ }^{5}$ In such schemes, the associated decrease policies could be referred to as linear loss dependent multiplicative decrease policies. It has been observed that such policies - examples are presented in $[10,29,18]$ - induce a smoother adaptation behavior and, thus, the loss dependent multiplicative decrease policies may be more appropriate for applications sensitive to large rate variation, such as the $\mathrm{CM}$ applications. In the approach described in the present paper, the introduced loss dependent multiplicative decrease policy (LDMD) uses $b_{\mathrm{D}}=d(1-f)$ and $a_{\mathrm{D}}=0$ in (19). The new rate shaping is based on the "current effective sending rate" - defined to be equal to $(1-f) \times$ current rate - scaled by a factor $d$ (new rate $=d \times$ current ef fective sending rate).

In [10] it is considered that $b_{\mathrm{D}}=(1-\beta f)$ and $a_{\mathrm{D}}=0$ in (19), where $\beta$ is a reduction parameter that may take a value between 2 and 5 with typical value $\beta=3$ and $f$ denotes the reported packet loss rate. In [29] the aforementioned decrease policy is included in a scheme referred to as the AI/PD scheme and is compared with the basic AI/MD scheme.

In [18] the next rate after a decrease is not determined by the current effective sending rate but by the moving average of the effective sending rate $\bar{R}^{6}$ multiplied by the factor $(1-d \beta)$, that is the new rate is given by $\bar{R}(1-d \beta)$. The constant $\beta$ has a very low value, e.g., 0.05 , and $d$ is a positive integer that may be exponentially increased (power of 2) depending on whether the current effective sending rate exceeds a

\footnotetext{
${ }^{5}$ Clearly, this is not the case of the basic AI/MD and binomial schemes.

${ }^{6}$ The average effective sending rate $\bar{R}$ is calculated as $\bar{R}=\mu \bar{R}+$ $(1-\mu) R$, where $\mu=0.875$ and $R$ the current effective sending rate given by $R=$ current rate $\times(1-f)$.
} 
threshold from its running average value.

Since in the LDMD policy the new rate is shaped and scaled based on the current effective sending rate, our approach appears to be more meaningful than the one in $[10,29]$ where the new rate is given by new rate $=(1-\beta f) \times$ current rate, which does not explicitly incorporate the scaled current effective sending rate. It is also simpler than that in [18], where the new rate, given by new rate $=\bar{R}(1-d \beta)$, is not based on the current effective sending rate but on the averaged rate $\bar{R}$. Furthermore, the simplicity of the proposed LDMD policy allows for a better understanding of the intrinsic properties of such a loss dependent decrease policy and result in properties not encountered in the other decrease policies. For instance, in the LDMD scheme ${ }^{7}$, the total load after a decrease action is independent from its prior value and equal to $d \times$ efficiency line, resulting in a regular adaptation behavior of the total load (see Propositions 5 and 6), a property not holding for other decrease policies. In this paper we show that the adaptation behavior is smoother, the flows converge faster to the fair share, the induced losses are lower, the throughput and network utilization is higher under the proposed DWAI/LDMD scheme than the AI/PD and LIMDH schemes.

Some theoretical work on congestion control schemes based on minimal and locally available information is presented in [24],[25],[26]. In [24] the potential of design networks that deliver low-loss, lowdelay services is demonstrated. The users adapt their rate using minimal and locally available information from the network, e.g., reported packet losses. In [25] the results of [24] are extended to (a) include random losses (e.g., produced in a wireless environment), and (b) use the explicit congestion notification (ECN) based marking mechanism in the routers instead of a dropping mechanism. In [26] the recent results on modeling, analysis and design of congestion control schemes for the Internet are surveyed. The work presented here is in line with the aforementioned results of [24-26] in the sense that minimal and locally available information, i.e., losses, is exploited by the proposed congestion control scheme.

\footnotetext{
${ }^{7}$ Under the assumptions of synchronized feedback and losses in proportion to the flow's rate.
}

\section{Conclusions}

In this paper a novel feedback-based congestion control scheme - the Distance Weighted Additive Increase (DWAI) and Loss Dependent Multiplicative Decrease (LDMD) scheme - is introduced and analyzed for flows whose rates are subject to a minimum and a maximum value constraint. Such flows could be those associated with streaming applications.

The innovative DWAI policy induces a typically diverse (for diverse flows' rates) per flow rate increase, unlike the basic AI policy: larger rate increment for flows that are at a larger distance from their maximum rate (self-adjusting characteristic). As a consequence we show that during rate increase phases the diverse rates tend to come closer in absolute terms and tend to the fair rate; this is not the case under the basic AI policy. As a result the proposed scheme is expected (and is shown in the presented results) to converge to fairness faster than the basic $\mathrm{AI} / \mathrm{MD}$ scheme. The introduced LDMD policy takes into consideration the induced packet loss rate when determining the rate decrement: it applies a large decrease factor under high losses helping overcome congestion quickly. Contrary to the LDMD policy, the basic MD policy cannot ensure such self-adjusting adaptation due to the associated fixed decrease factor.

The proposed scheme has been studied under the assumption of synchronized feedback and packet losses proportional to the flow's rate. The convergence to fairness and efficiency have been shown while the detailed oscillatory adaptation behavior has been examined and determined. Meaningful comparisons with the behavior under the basic AI/MD scheme have been conducted shedding more light into the intrinsic behaviors of both schemes and the better behavior of the DWAI/LDMD scheme with respect to smoothness, convergence to fairness and packet losses. The latter have also been illustrated in the presented simulation results under the environment assumed in the study, as well as in an environment in which the feedback is not synchronized, the flows are initiated at different times, and present different round trip times, by conducting ns-2-based simulations. We show in the simulation results the better behavior of the DWAI/LDMD scheme against the non-AI/MD schemes AI/PD, LIMDH, and the TCPcompatible schemes TFRC and IIAD. 


\section{REFERENCES}

1. V. Jacobson, Congestion avoidance control. In Proceedings of the SIGCOMM '88 Conference on Communications Architectures and Protocols (1988).

2. RFC-2001. TCP Slow Start, Congestion Avoidance, Fast Retransmit, and Fast Recovery Algorithms.

3. D.M Chiu, R. Jain, Analysis of the Increase and Decrease Algorithms for Congestion Avoidance in Computer Networks, Computer Networks and ISDN Systems 17 (1989) 1-14.

4. J. Padhye, V. Firoiu, D. Towsley, J.Kurose, Modeling TCP Throughput: A Simple Model and its Empirical Validation, ACM SIGCOMM'98.

5. RFC 1889. RTP: A Transport Protocol for RealTime Applications.

6. Floyd, S., and Fall, K., Promoting the Use of End-to-End Congestion Control in the Internet. IEEE/ACM Transactions on Networking. August 1999.

7. S. Schenker, Fundamental Design Issues for the Future Internet. IEEE Journal on Selected Areas in Communications, Vol. 13, No. 7, September 1995.

8. I. Busse, B. Defner, H. Schulzrinne, Dynamic QoS Control of Multimedia Application based on RTP. May 1995.

9. J. Bolot,T. Turletti, Experience with Rate Control Mechanisms for Packet Video in the Internet. ACM SIGCOMM Computer Communication Review, Vol. 28, No 1, pp. 4-15, Jan. 1998.

10. D. Sisalem, F. Emanuel, H. Schulzrinne, The Loss-Delay Based Adjustment Algorithm: A TCP-Friendly Adaptation Scheme. 1998.

11. D. Sisalem and A. Wolisz, LDA+: Comparison with RAP, TFRCP. IEEE International Conference on Multimedia (ICME 2000), July 30 - August 2, 2000, New York.

12. D. Sisalem and A. Wolisz, MLDA: A TCPfriendly congestion control framework for heterogeneous multicast environments. Eighth International Workshop on Quality of Service (IWQoS 2000), 5-7 June 2000, Pittsburgh.

13. D. Sisalem and A. Wolisz, Constrained TCPfriendly congestion control for multimedia communication tech. rep., GMD Fokus, Berlin Ger- many, Feb. 2000.

14. D. Sisalem, A. Wolisz, Towards TCP-Friendly Adaptive Multimedia Applications Based on RTP. Fourth IEEE Symposium on Computers and Communications (ISCC'1999), (Red Sea, Egypt), July 1999.

15. R. Rejaie, M. Handley, D. Estrin, An End-toend Rate-based Congestion Control Mechanism for Realtime Streams in the Internet. Proc. INFOCOM 99, 1999.

16. J. Padhye, J. Kurose, D. Towsley, R. Koodli, A Model Based TCP-Friendly Rate Control Protocol. Proc. IEEE NOSSDAV'99 (Basking Ridge, NJ, June 1999).

17. RFC 2475. An Architecture for Differentiated Services. December 1998.

18. T. Kim, S. Lu and V. Bharghavan, Improving Congestion Control Performance Through Loss Differentiation. International Conference on Computers and Communications Networks '99, Boston, MA. October 1999.

19. P. Balaouras, I. Stavrakakis, A Multi-state Congestion Control Scheme for Unicast Continuous Media Flows, Evolutionary Trends of the Internet, 2001 International Workshop on Digital Communications, IWDC 2001, Taormina, Italy, Proceedings, LNCS 2170 (2001), Springer.

20. D. Bansal, H. Balakrishnan, Binomial Congestion Control Algorithms, IEEE INFOCOM 2001.

21. D. Tan, A. Zakhor, Real-time Internet video using error resilient scalable compression and TCP-friendly transport protocol. IEEE/ACM Transactions on Multimedia, May 1999.

22. D. Loguinov, H. Radha, Increase-Decrease Congestion Control for Real-time Streaming: Scalability. IEEE INFOCOM, June 2002.

23. S. Floyd, J. Padhye, Joerg Widmer, Equationbased Congestion Control for Unicast Applications. ACM SIGCOMM '00.

24. F.P. Kelly, A. Maulloo, and D. Tan, Rate control in communication networks: shadow prices, proportional fairness and stability. Journal of the Operational Research Society, 49, 1998.

25. S. Kunniyur, R. Srikant, End-to-End Congestion Control Schemes: Utility Functions, Random Losses and ECN Marks. IEEE INFOCOM, 2000.

26. S.H. Low, R. Srikant, A Mathematical Framework for Designing a Low-Loss, 
Low-Delay Internet. Networks and Spatial Economics, special issue on "Crossovers between Transportation Planning and Telecommunications", January-February 2003, http://netlab.caltech.edu/pub/papers/LowSrikant03.pdf

27. S. Jin. L. Guo, I. Matta and A. Bestavros, TCPfriendly SIMD congestion control and its convergence behavior, in Proc. Int. Conf, Network Protocols (ICNP), Nov. 2001, pp. 156-164.

28. S. Jin. L. Guo, I. Matta and A. Bestavros, A Spectrum of TCP-Friendly window-based Congestion Control Algorithms. IEEE/ACM Transactions on Networking, Vol. 11, No. 3, June 2003.

29. K. Lee, T. Kim, V. Bharghavan A Comparison of Two Popular End-to-End Congestion Control Algorithms: The Case of AIMD and AIPD. University of Illinois Technical Report http://timely.crhc.uiuc.edu/kwlee/psfiles/infocom2001.ps

30. M. Reisslein, et al. Traffic and Quality Characterization of Scalable Encoded Video: A Large-Scale Trace-Based Study Part 2: Statistical Analysis of Single-Layer Encoded Video. http://www.eas.asu.edu/trace

31. ITU-T Rec. H.323, Visual Telephone Systems and Terminal Equipment for Local Area Networks which Provide a Non-Guaranteed Quality of Service, 1996. 